\title{
LHC phenomenology of the type II seesaw mechanism: Observability of neutral scalars in the nondegenerate case
}

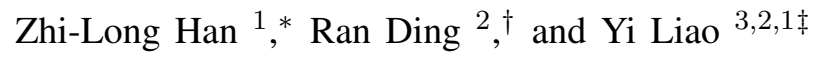 \\ ${ }^{1}$ School of Physics, Nankai University, Tianjin 300071, China \\ ${ }^{2}$ Center for High Energy Physics, Peking University, Beijing 100871, China \\ ${ }^{3}$ State Key Laboratory of Theoretical Physics, Institute of Theoretical Physics, \\ Chinese Academy of Sciences, Beijing 100190, China
}

(Dated: July 4, 2018)

\begin{abstract}
This is a sequel to our previous work on LHC phenomenology of the type II seesaw model in the nondegenerate case. In this work, we further study the pair and associated production of the neutral scalars $H^{0} / A^{0}$. We restrict ourselves to the so-called negative scenario characterized by the mass order $M_{H^{ \pm \pm}}>M_{H^{ \pm}}>M_{H^{0} / A^{0}}$, in which the $H^{0} / A^{0}$ production receives significant enhancement from cascade decays of the charged scalars $H^{ \pm \pm}, H^{ \pm}$. We consider three important signal channels- $b \bar{b} \gamma \gamma, b \bar{b} \tau^{+} \tau^{-}, b \bar{b} \ell^{+} \ell^{-} E_{T}$-and perform detailed simulations. We find that at the $14 \mathrm{TeV}$ LHC with an integrated luminosity of $3000 \mathrm{fb}^{-1}$, a $5 \sigma$ mass reach of 151,150 , and $180 \mathrm{GeV}$, respectively, is possible in the three channels from the pure Drell-Yan $H^{0} A^{0}$ production, while the cascade-decay-enhanced $H^{0} / A^{0}$ production can push the mass limit further to 164,177 , and $200 \mathrm{GeV}$. The neutral scalars in the negative scenario are thus accessible at LHC run II.
\end{abstract}

* hanzhilong@mail.nankai.edu.cn

† dingran@mail.nankai.edu.cn

$\ddagger$ liaoy@ nankai.edu.cn 


\section{INTRODUCTION}

In a previous paper [1], we presented a comprehensive analysis on the LHC signatures of the type II seesaw model of neutrino masses in the nondegenerate case of the triplet scalars. In this companion paper, another important signature - the pair and associated production of the neutral scalars-is explored in great detail. This is correlated to the pair production of the standard model (SM) Higgs boson, $h$, which has attracted lots of theoretical and experimental interest [2, 3] since its discovery [4, 5], because the pair production can be used to gain information on the electroweak symmetry breaking sector [6]. Since any new ingredients in the scalar sector can potentially alter the production and decay properties of the Higgs boson, a thorough examination of the properties offers a diagnostic tool to physics effects beyond the SM. The Higgs boson pair production has been well studied for collider phenomenology in the framework of the SM and beyond [6-22], and extensively studied in various new physics models [23-47], as well as in the effective field theory approach of anomalous couplings [48-51] and effective operators [52-56].

The pair production of the SM Higgs boson proceeds dominantly through the gluon fusion process [6, 8], and has a cross section at the $14 \mathrm{TeV}$ LHC (LHC14) of about $18 \mathrm{fb}$ at leading order [6]. 1 It can be utilized to measure the Higgs trilinear coupling. A series of studies have surveyed its observability in the $b \bar{b} \gamma \gamma$, $b \bar{b} \tau^{+} \tau^{-}, b \bar{b} W^{+} W^{-}, b \bar{b} b \bar{b}$, and $W W^{*} W W^{*}$ signal channels [11-13, 21, 22, 36, 57]. For the theoretical and experimental status of the Higgs trilinear coupling and pair production at the LHC, see Refs. [57, 58]. In summary, at the $14 \mathrm{TeV}$ LHC with an integrated luminosity of $3000 \mathrm{fb}^{-1}$ (LHC14@3000), the trilinear coupling could be measured at an accuracy of $\sim 40 \%$ [18], and thus leaves potential space for new physics.

As we pointed out in Ref. [1], in the negative scenario of the type II seesaw model where the doubly charged scalars $H^{ \pm \pm}$are the heaviest and the neutral ones $H^{0} / A^{0}$ the lightest, i.e., $M_{H^{ \pm \pm}}>M_{H^{ \pm}}>$ $M_{H^{0} / A^{0}}$, the associated $H^{0} A^{0}$ production gives the same signals as the SM Higgs pair production while enjoying a larger cross section. The leading production channel is the Drell-Yan process $p p \rightarrow Z^{*} \rightarrow$ $H^{0} A^{0}$, with a typical cross section $20-500 \mathrm{fb}$ in the mass region $130-300 \mathrm{GeV}$. Additionally, there exists a sizable enhancement from the cascade decays of the heavier charged scalars, which also gives some indirect evidence for these particles. The purpose of this paper is to examine the importance of the $H^{0} A^{0}$ production with an emphasis on the contribution from cascade decays and to explore their observability.

The paper is organized as follows. In Sec. II] we summarize the relevant part of the type II seesaw and explore the decay properties of $H^{0}, A^{0}$ in the negative scenario. Sections III and IV]contain our systematical analysis of the impact of cascade decays on the $H^{0} / A^{0}$ production in the three signal channels, $b \bar{b} \gamma \gamma$, $b \bar{b} \tau^{+} \tau^{-}$, and $b \bar{b} \ell^{+} \ell^{-} E_{T}$. We discuss the observability of the signals and estimate the required integrated

\footnotetext{
${ }^{1}$ This number is modified to $33 \mathrm{fb}$ at next-to-leading order [7] and to $40 \mathrm{fb}$ at next-to-next-to-leading order [16].
} 
luminosity for a certain mass reach and significance. Discussions and conclusions are presented in Sec. $\mathrm{V}$ In most cases, we will follow the notations and conventions in Ref. [1].

\section{DECAY PROPERTIES OF NEUTRAL SCALARS IN THE NEGATIVE SCENARIO}

The type II seesaw and its various experimental constraints have been reviewed in our previous work [1]. Here we recall the most relevant content that is necessary for our study of the decay properties of the scalars in this section and of their detection at the LHC in later sections.

The type II seesaw model introduces an extra scalar triplet $\Delta$ of hypercharge two [59] on top of the SM Higgs doublet $\Phi$ of hypercharge unity. Writing $\Delta$ in matrix form, the most general scalar potential is

$$
\begin{aligned}
V(\Phi, \Delta)= & m^{2} \Phi^{\dagger} \Phi+M^{2} \operatorname{Tr}\left(\Delta^{\dagger} \Delta\right)+\lambda_{1}\left(\Phi^{\dagger} \Phi\right)^{2}+\lambda_{2}\left(\operatorname{Tr}\left(\Delta^{\dagger} \Delta\right)\right)^{2}+\lambda_{3} \operatorname{Tr}\left(\Delta^{\dagger} \Delta\right)^{2} \\
& +\lambda_{4}\left(\Phi^{\dagger} \Phi\right) \operatorname{Tr}\left(\Delta^{\dagger} \Delta\right)+\lambda_{5} \Phi^{\dagger} \Delta \Delta^{\dagger} \Phi+\left(\mu \Phi^{T} i \tau^{2} \Delta^{\dagger} \Phi+\text { H.c. }\right)
\end{aligned}
$$

As in the SM, $m^{2}<0$ is assumed to trigger spontaneous symmetry breaking, while $M^{2}>0$ sets the mass scale of the new scalars. The vacuum expectation value (vev) $v$ of $\Phi$ then induces via the $\mu$ term a vev $v_{\Delta}$ for $\Delta$. The components of equal charge (and also of identical $C P$ in the case of neutral components) in $\Delta$ and $\Phi$ then mix into physical scalars $H^{ \pm} ; A^{0} ; H^{0}, h$ and would-be Goldstone bosons $G^{ \pm ; 0}$, with the mixing angles specified by (see, for instance, Refs. [60, 61])

$$
\tan \theta_{+}=\frac{\sqrt{2} v_{\Delta}}{v}, \tan \alpha=\frac{2 v_{\Delta}}{v}, \tan 2 \theta_{0}=\frac{2 v_{\Delta}}{v} \frac{v^{2}\left(\lambda_{4}+\lambda_{5}\right)-2 M_{\Delta}^{2}}{2 v^{2} \lambda_{1}-M_{\Delta}^{2}-v_{\Delta}^{2}\left(\lambda_{2}+\lambda_{3}\right)},
$$

where an auxiliary parameter is introduced for convenience,

$$
M_{\Delta}^{2}=\frac{v^{2} \mu}{\sqrt{2} v_{\Delta}}
$$

To a good approximation, the SM-like Higgs boson $h$ has the mass $M_{h} \approx \sqrt{2 \lambda_{1}} v$, the new neutral scalars $H^{0}, A^{0}$ have an equal mass $M_{H^{0}} \approx M_{A^{0}} \approx M_{\Delta}$, and the new scalars of various charges are equidistant in squared masses:

$$
M_{H^{ \pm \pm}}^{2}-M_{H^{ \pm}}^{2} \approx M_{H^{ \pm}}^{2}-M_{H^{0} / A^{0}}^{2} \approx-\frac{1}{4} \lambda_{5} v^{2}
$$

There are thus two scenarios of spectra, positive or negative, according to the sign of $\lambda_{5}$. For convenience, we define $\Delta M \equiv M_{H^{ \pm}}-M_{H^{0}} / A^{0}$.

In the rest of this section, we discuss the decay properties of the new scalars in the negative scenario with an emphasis on $H^{0}$ and $A^{0}$. The explicit expressions for the relevant decay widths can be found in Refs. [62 64]. It has been shown that $H^{0} / A^{0}$ decays dominantly into neutrinos for $v_{\Delta}<10^{-4} \mathrm{GeV}$ [65], 

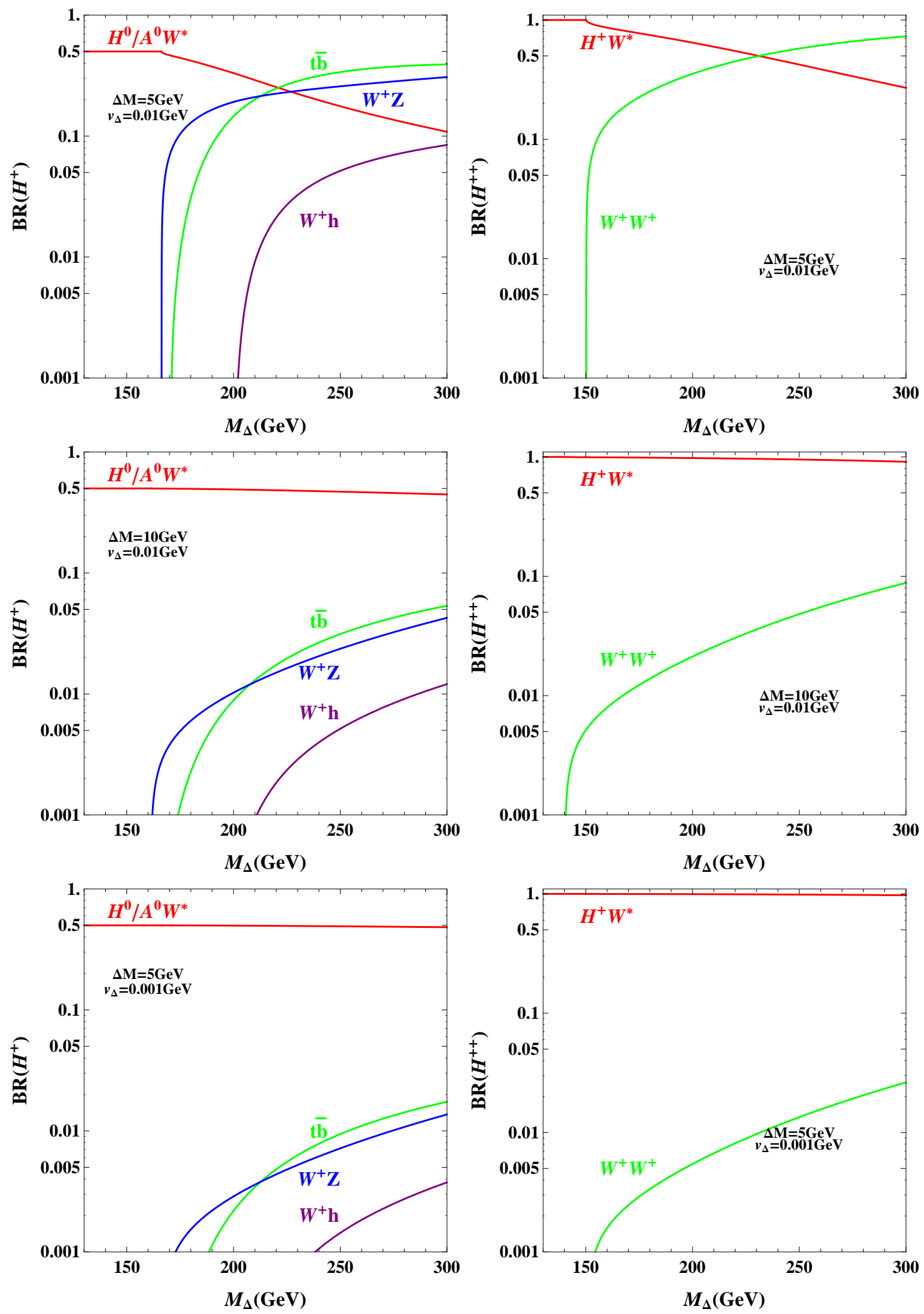

FIG. 1. Branching ratios of $H^{ \pm}$and $H^{ \pm \pm}$versus $M_{\Delta}$ at some benchmark points of $\Delta M$ and $v_{\Delta}:\left(\Delta M, v_{\Delta}\right)=$ $(5,0.01),(10,0.01),(5,0.001) \mathrm{GeV}$, from the upper to the lower panels. 
resulting in totally invisible final states. We will restrict ourselves to $v_{\Delta} \gg 10^{-4} \mathrm{GeV}$ in this work, where $H^{0} / A^{0}$ dominantly decays into visible particles. Before we detail their decay properties, we give a brief account of the cascade decays of the charged scalars. The branching ratios of the cascade decays are controlled by the three parameters, $v_{\Delta}, \Delta M$, and $M_{\Delta}$. The cascade decays dominate in the moderate region of $v_{\Delta}$ and for $\Delta M$ not too small, where a minimum value of $\Delta M \sim 2 \mathrm{GeV}$ appears around $v_{\Delta} \sim 10^{-4} \mathrm{GeV}[1,62,65,66]$. In Fig. 1, the branching ratios of $H^{ \pm}$and $H^{ \pm \pm}$are shown as a function of $M_{\Delta}$ at some benchmark points of $v_{\Delta}$ and $\Delta M$. Basically speaking, in the mass region $M_{\Delta}=130$ $300 \mathrm{GeV}$, the cascade decays are dominant for a relatively large mass splitting $\Delta M$ (as shown in the middle panel of Fig. 1) or a relatively small $v_{\Delta}$ (in the lower panel).

\section{A. $H^{0}$ decays}

At tree level, $H^{0}$ can decay to $f \bar{f}(f=q, l), \nu \nu, W^{+} W^{-}, Z Z$, and $h h$. It can also decay to $g g, \gamma \gamma$, and $Z \gamma$ through radiative effects. Similarly, $A^{0} \rightarrow f \bar{f}, \nu \nu, Z h$ at tree level, and it has the same decay modes as $H^{0}$ at the loop level. Since we have chosen $v_{\Delta} \gg 10^{-4} \mathrm{GeV}$, the neutrino mode can be safely neglected for both $H^{0}$ and $A^{0}$. Previous work usually concentrated on the decoupling region where the neutral scalars $H^{0} / A^{0}$ are much heaver than the light $C P$-even Higgs $h$ and the scalar self-couplings $\lambda_{i}$ are taken to be zero for simplicity [65]. In this case, the mixing angle $\theta_{0} \approx \alpha$, and the $H^{0} W^{+} W^{-}$coupling [being proportional to $\sin \left(\alpha-\theta_{0}\right)$ ] tends to vanish. As a consequence, the $W$-pair mode is absent and the dominant channels are $H^{0} \rightarrow h h, Z Z$ for a heavy $H^{0}$. In contrast, we take into account the effect of scalar self-interactions and focus on the nondecoupling regime, i.e., $H^{0} / A^{0}$ are not much heavier than $h$.

For illustration, we choose the benchmark values $v_{\Delta}=10^{-3} \mathrm{GeV}, \Delta M=5 \mathrm{GeV}$; then, $\lambda_{5}$ is determined by Eq. (4) upon specifying $M_{\Delta}$. 2 To investigate the effect of the scalar self-interactions, we note the following features in the decays of $H^{0}$. 1) The decay widths of $H^{0} \rightarrow f \bar{f}, g g$ differ from those of $h$ only by a factor of $\sin ^{2} \theta_{0}$, which leads to similar behavior for $H^{0}$ and $h$. 2) The only free parameter for the mixing between $H^{0}$ and $h$ is $\lambda_{4}$, because [as shown in Eq. [2] ] the impact of $\lambda_{2,3}$ is suppressed by a small $v_{\Delta}$ and a relatively large mass difference between $M_{\Delta}$ and $M_{h}$ while $\lambda_{1}$ is fixed by $M_{h}$. 3) $\lambda_{4}$ enters the $H^{0} W^{+} W^{-}$and $H^{0} Z Z$ couplings and thus affects the decays $H^{0} \rightarrow W^{+} W^{-}, Z Z$. 4) The $H^{0} h h$ coupling simplifies for $v_{\Delta} \ll v$ such that the only free parameter in the decay $H^{0} \rightarrow h h$ is again $\lambda_{4}$. As a consequence of these features, we shall choose $\lambda_{4}$ as a free parameter and vary it in the range $[-1.0,1.0]$, and fix the couplings $\lambda_{2}=\lambda_{3}=0.1$ which are involved in loop-induced decays.

We first examine the branching ratios of $H^{0} \rightarrow f \bar{f}$. $\mathrm{BR}\left(H^{0} \rightarrow b \bar{b}\right)$ and $\mathrm{BR}\left(H^{0} \rightarrow t \bar{t}\right)$ are plotted in

\footnotetext{
${ }^{2}$ As pointed out in Ref. [62], varying $v_{\Delta}$ in the range $10^{-3}-1 \mathrm{GeV}$ would not change the branching ratios significantly.
} 

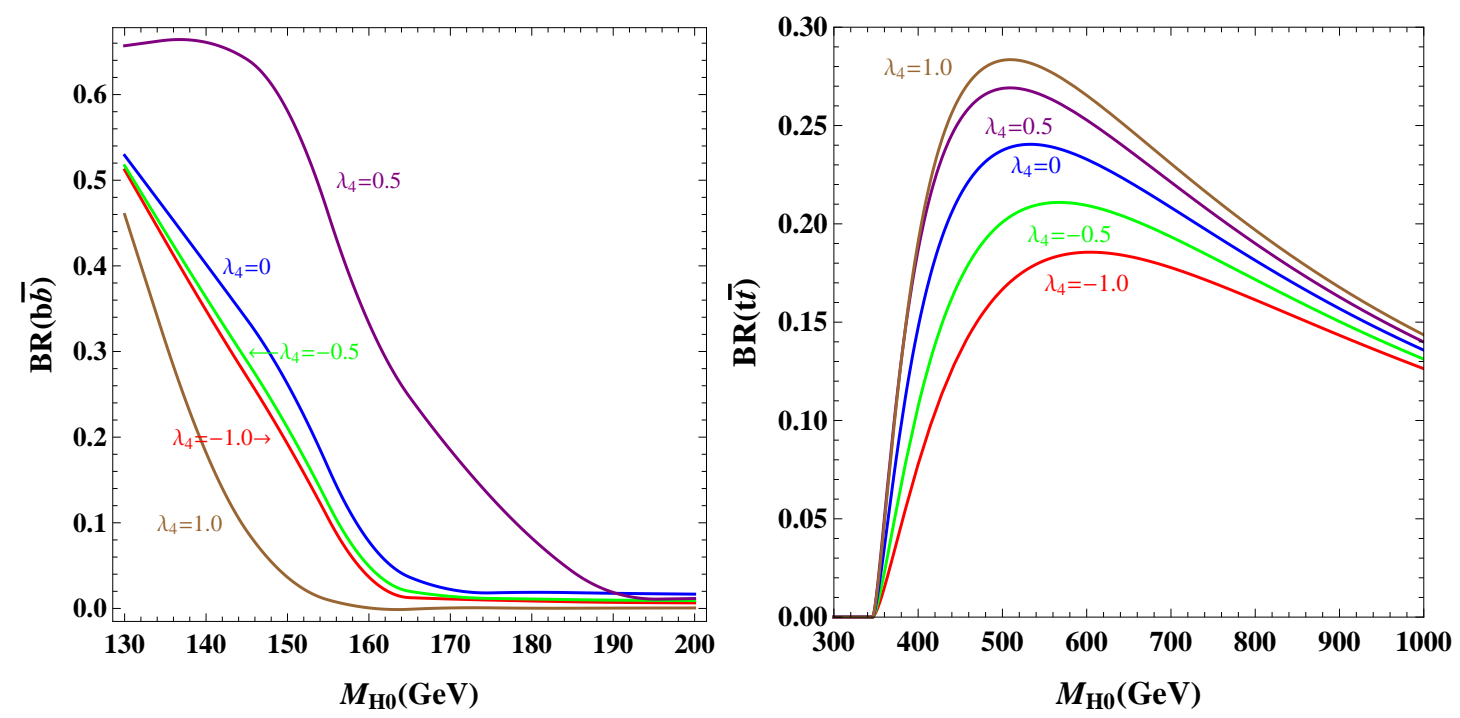

FIG. 2. Branching ratios of $H^{0} \rightarrow b \bar{b}$ and $H^{0} \rightarrow t \bar{t}$ as a function of $M_{H^{0}}$ for various values of $\lambda_{4}$.

Fig. 2 for different mass regions of $H^{0}$. 3 It is clear that the variation of $\mathrm{BR}\left(H^{0} \rightarrow b \bar{b}\right)$ is more dramatic for $\lambda_{4}>0$. The maximum of $\operatorname{BR}\left(H^{0} \rightarrow b \bar{b}\right)$ appears at $\lambda_{4} \approx 0.5$. Obviously, $\operatorname{BR}\left(H^{0} \rightarrow b \bar{b}\right)$ is a nonmonotonic function of $\lambda_{4}$, while $\operatorname{BR}\left(H^{0} \rightarrow t \bar{t}\right)$ monotonically increases with $\lambda_{4}$. As will be discussed later, this different behavior in the two mass regions is due mainly to a zero in the $H^{0} Z Z$ coupling.

Now we study the bosonic decays $H^{0} \rightarrow W^{+} W^{-}, Z Z$, hh. In the left panel of Fig. 3, we present the branching ratios of $H^{0} \rightarrow W^{+} W^{-}, Z Z$ in the mass region 130-300 GeV. For most values of $\lambda_{4}$, $\mathrm{BR}\left(H^{0} \rightarrow W^{+} W^{-}\right)$increases with $M_{H^{0}}$ when $M_{H^{0}}<2 M_{W}$, and varying $\lambda_{4}$ for $\lambda_{4}>0$ changes it considerably. $\lambda_{4}$ has a strong impact on $\mathrm{BR}\left(H^{0} \rightarrow W^{+} W^{-}\right)$in the mass region $2 M_{Z}<M_{H^{0}}<2 M_{h}$ where the decay channel dominates overwhelmingly for $\lambda_{4}<0$ but becomes negligible for $\lambda_{4}$ approaching about 0.5 . However, once the $H^{0} \rightarrow h h$ channel is opened, $H^{0} \rightarrow W^{+} W^{-}$is suppressed significantly independent of $\lambda_{4}$. The decay $H^{0} \rightarrow Z Z$ cannot dominate when $M_{H^{0}}<2 M_{W}$. In the mass region $2 M_{Z}<$ $M_{H^{0}}<2 M_{h}$, it is complementary with the $W^{+} W^{-}$channel, so their behavior is just opposite. More interestingly, there is a zero point for the $H^{0} Z Z$ coupling, which is proportional to $\left(v \sin \theta_{0}-4 v_{\Delta} \cos \theta_{0}\right)$. According to Eq. (2), one obtains the corresponding $M_{\Delta}$ at the zero:

$$
M_{\Delta}^{0}(Z Z)=\sqrt{2 M_{h}^{2}-\frac{1}{2}\left(\lambda_{4}+\lambda_{5}\right) v^{2}} .
$$

Note that the above relation only holds for $\lambda_{4}+\lambda_{5}<2 M_{h}^{2} / v^{2} \approx 0.5$, since we are working in the scenario where $M_{\Delta}>M_{h}$. The existence of the zero coupling explains the presence of the nodes in $\operatorname{BR}\left(H^{0} \rightarrow Z Z\right)$ for $\lambda_{4} \leq 0$.

\footnotetext{
${ }^{3}$ The influence of $\lambda_{4}$ for light fermions $b, c, \tau, \mu$ and gluons is similar, so we only present $\mathrm{BR}\left(H^{0} \rightarrow b \bar{b}\right)$ in Fig. 2
} 

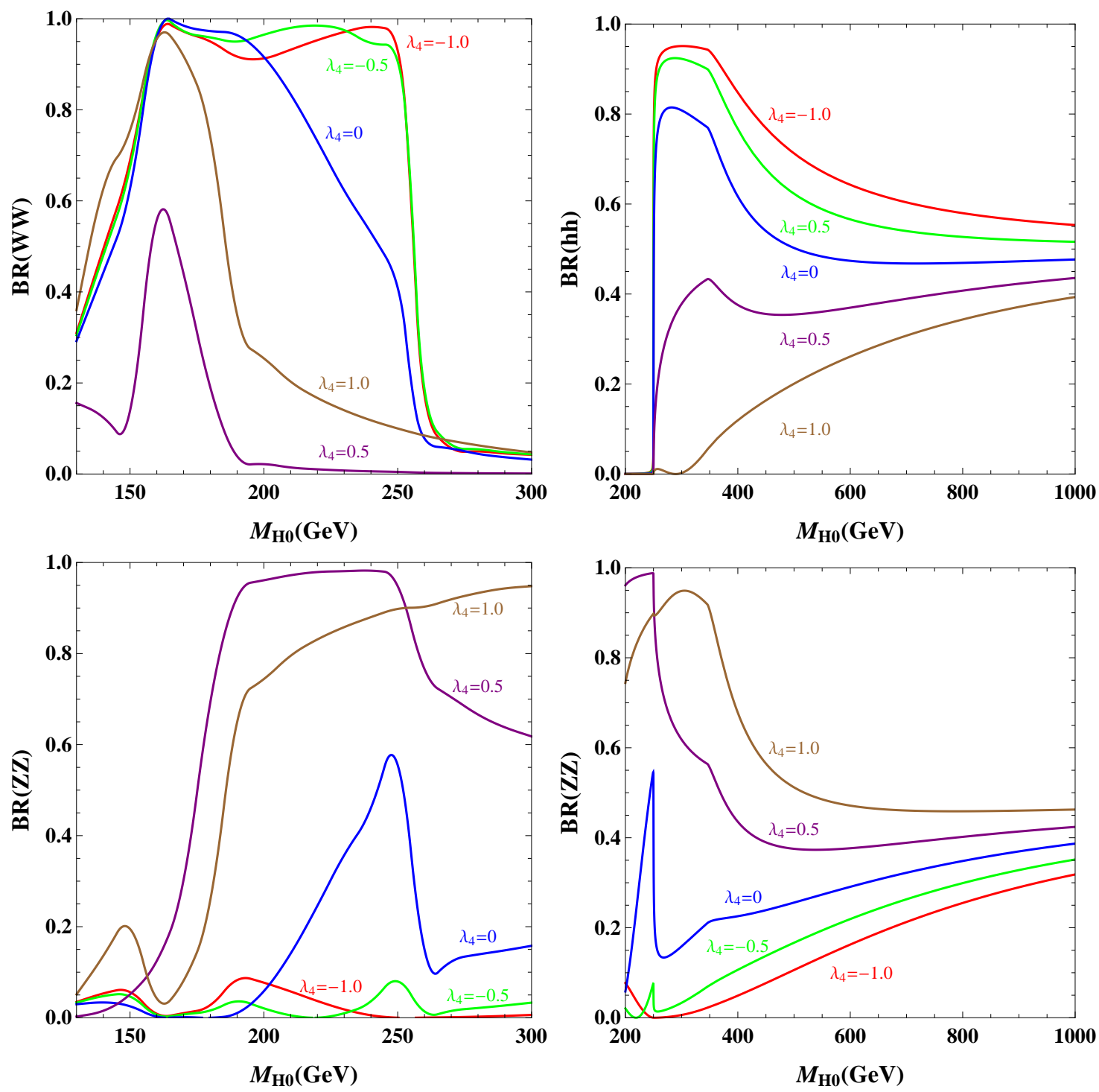

FIG. 3. Left: Branching ratios of $H^{0} \rightarrow W^{+} W^{-}, Z Z$ as a function of $M_{H^{0}}$ in the mass region $130-300 \mathrm{GeV}$. Right: Branching ratios of $H^{0} \rightarrow h h, Z Z$ as a function of $M_{H^{0}}$ in the mass region $200-1000 \mathrm{GeV}$.

In the right panel of Fig. 3, $\mathrm{BR}\left(H^{0} \rightarrow h h, Z Z\right)$ are shown in the mass region $200-1000 \mathrm{GeV}$. When $M_{H^{0}}>2 M_{h}$, the dependence on $\lambda_{4}$ is simple: a larger $\lambda_{4}$ corresponds to a smaller $\operatorname{BR}\left(H^{0} \rightarrow h h\right)$ and a larger $\mathrm{BR}\left(H^{0} \rightarrow Z Z\right)$. It is clear that $\lambda_{4}$ has a more significant impact in the mass region $200 \sim 350 \mathrm{GeV}$, and varying $\lambda_{4}$ could change $\mathrm{BR}\left(H^{0} \rightarrow Z Z\right)$ from 0 to 0.9 . Once $M_{H^{0}}$ exceeds $2 M_{t}$, the evolution of $\operatorname{Br}\left(H^{0} \rightarrow h h, Z Z\right)$ becomes smooth with the increase of $M_{H^{0}}$. There also exists a zero point for the $H^{0} h h$ coupling, which can be obtained as for the $Z Z$ channel:

$$
M_{\Delta}^{0}(h h)=\sqrt{2\left(\lambda_{4}+\lambda_{5}\right) v^{2}-2 M_{h}^{2}},
$$



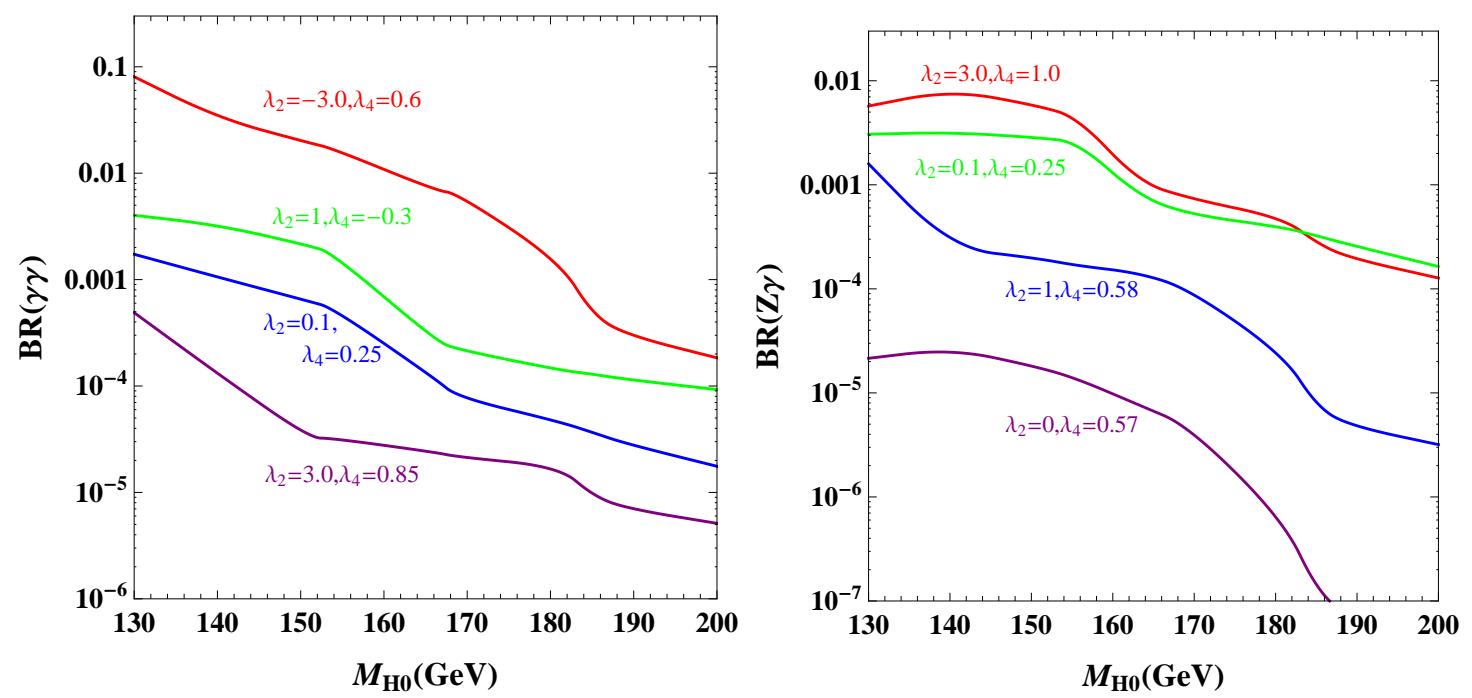

FIG. 4. Branching ratios of $H^{0} \rightarrow \gamma \gamma, Z \gamma$ as a function of $M_{H^{0}}$ for various sets of $\lambda_{2,4}$ values.

which is valid for $\lambda_{4}+\lambda_{5}>3 M_{h}^{2} / 2 v^{2} \approx 0.375$.

Finally, we investigate the loop-induced decays, $H^{0} \rightarrow \gamma \gamma, Z \gamma$. In addition to the usual contributions from the top quark and $W$ boson, the new charged scalars $H^{ \pm}$and $H^{ \pm \pm}$also contribute to the decays. These new terms involve the $H^{0} H^{+} H^{-}$and $H^{0} H^{++} H^{--}$couplings, which are proportional to

$$
\begin{aligned}
H^{0} H^{+} H^{-} & :\left[\left(2 \lambda_{2}+2 \lambda_{3}-\lambda_{5}\right) \sin \alpha \cos \theta_{0}-\left(2 \lambda_{4}+\lambda_{5}\right) \cos \alpha \sin \theta_{0}\right], \\
H^{0} H^{++} H^{--} & :\left(\lambda_{2} \sin \alpha \cos \theta_{0}-\lambda_{4} \cos \alpha \sin \theta_{0}\right) .
\end{aligned}
$$

One therefore has to consider the scalar self-couplings $\lambda_{2,3}$. For simplicity, we set $\lambda_{2}=\lambda_{3}$ and vary them from -3.0 to 3.0. In Fig. 4, we display $\mathrm{BR}\left(H^{0} \rightarrow \gamma \gamma\right)$ and $\mathrm{BR}\left(H^{0} \rightarrow Z \gamma\right)$ versus $M_{H^{0}}$ for some typical sets of $\lambda_{2,4}$ values. The evolution of both branching ratios crosses 3 orders of magnitude in this parameter region. The resulting enhancement compared with $h \rightarrow \gamma \gamma$ in the SM looks significant: the maximal enhancement can be achieved at the level of $9 \%$ for the $H^{0} \rightarrow \gamma \gamma$ channel at $M_{H^{0}}=130 \mathrm{GeV}$, and of $0.7 \%$ for the $H^{0} \rightarrow Z \gamma$ channel at $M_{H^{0}} \approx 140 \mathrm{GeV}$.

\section{B. $A^{0}$ decays}

Similar to $H^{0}$, the decay widths of $A^{0} \rightarrow f \bar{f}, g g$ differ from those of $h$ by a factor of $\sin ^{2} \alpha$ with $\alpha$ being given in Eq. 22. Moreover, the only vertex which involves $\lambda_{i}$ is the $A^{0} Z h$ coupling proportional to $\left(\cos \theta_{0} \sin \alpha-2 \sin \theta_{0} \cos \alpha\right)$. As a consequence, one can only choose $\lambda_{4}$ as a free parameter to illustrate the influence of scalar interactions. In this section, we also vary $\lambda_{4}$ from -1.0 to 1.0 and take the same benchmark values for $v_{\Delta}$ and $\Delta M$ as for the $H^{0}$ decays. 

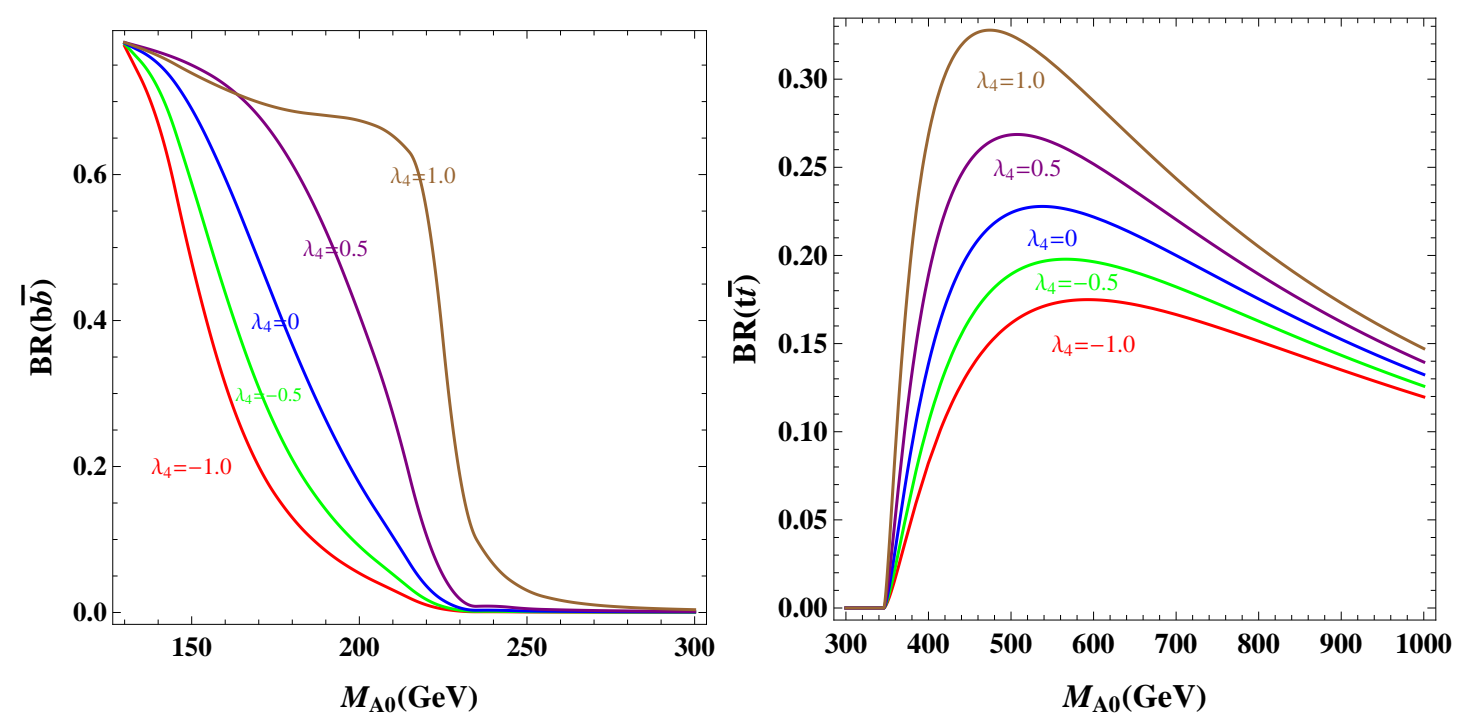

FIG. 5. Branching ratios of $A^{0} \rightarrow b \bar{b}, t \bar{t}$ as a function of $M_{A^{0}}$ for various values of $\lambda_{4}$.

In the left panel of Fig. 5, we present $\operatorname{BR}\left(A^{0} \rightarrow b \bar{b}\right)$ as a function of $M_{A^{0}}$. $4^{4}$ For a fixed value of $\lambda_{4}$, $\mathrm{BR}\left(A^{0} \rightarrow b \bar{b}\right)$ decreases as $M_{A^{0}}$ increases. The dependence of $\mathrm{BR}\left(A^{0} \rightarrow b \bar{b}\right)$ on $\lambda_{4}$ is simple: The larger $\lambda_{4}$ is, the larger $\mathrm{BR}\left(A^{0} \rightarrow b \bar{b}\right)$ is. And $\mathrm{BR}\left(A^{0} \rightarrow b \bar{b}\right)$ can be dominant with $\lambda_{4}=1.0$ as long as $A^{0} \rightarrow Z h$ is not fully opened. The right panel of Fig. 5 shows $\operatorname{BR}\left(A^{0} \rightarrow t \bar{t}\right)$, which is very similar to $\operatorname{BR}\left(H^{0} \rightarrow t \bar{t}\right)$.
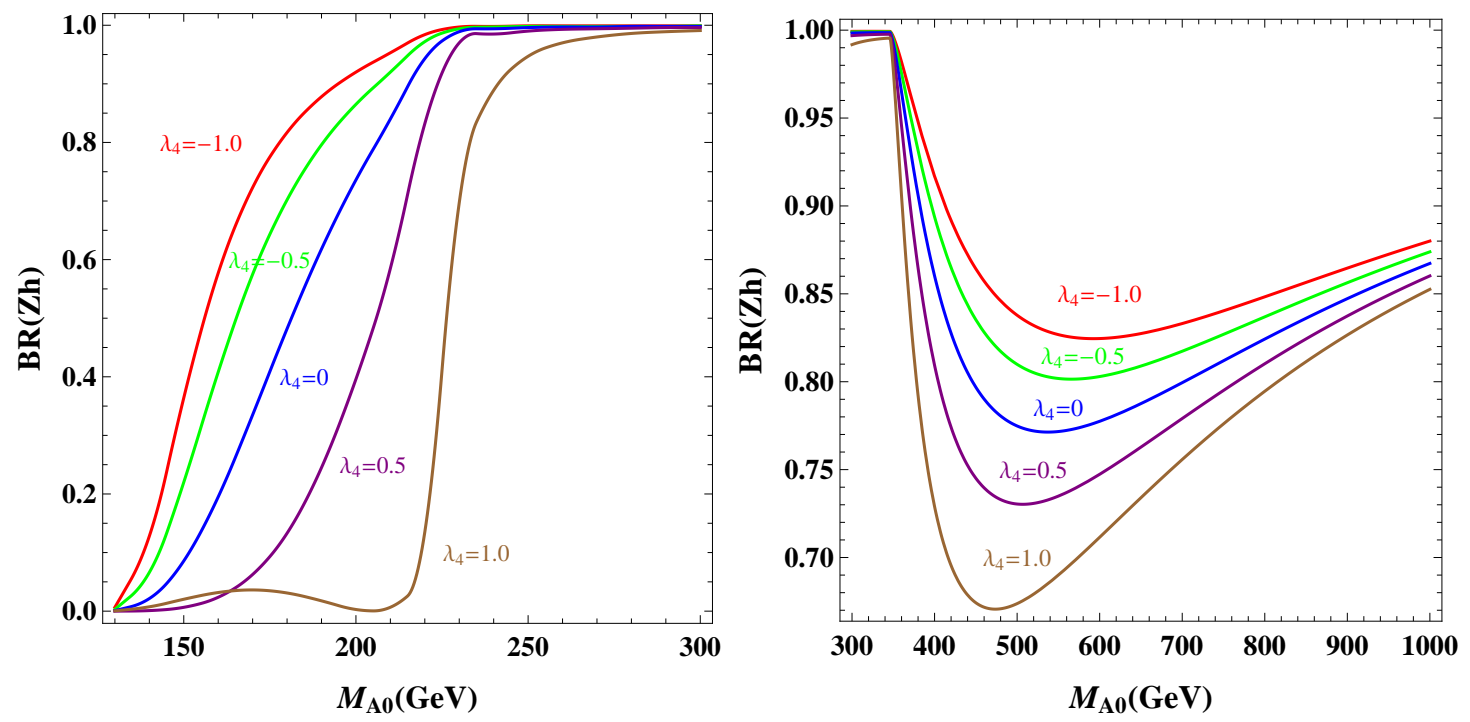

FIG. 6. Branching ratios of $A^{0} \rightarrow Z h$ as a function of $M_{A^{0}}$ for various values of $\lambda_{4}$..

We then study the most important decay $A^{0} \rightarrow Z h$. In Fig. 6, we present $\mathrm{BR}\left(A^{0} \rightarrow Z h\right)$ as a function

\footnotetext{
${ }^{4}$ As before, the influence of $\lambda_{4}$ on the $A^{0} \rightarrow f \bar{f}, g g$ channels is similar to the $b \bar{b}$ mode.
} 

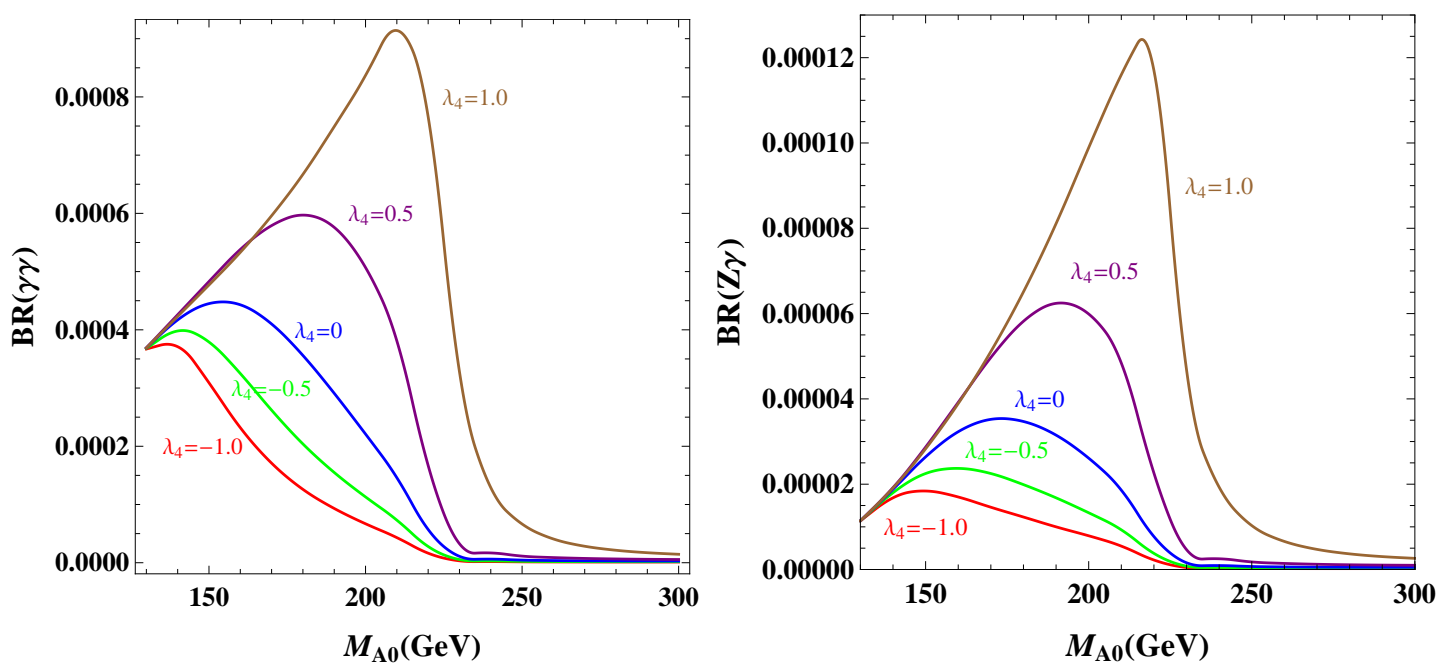

FIG. 7. Branching ratios of $A^{0} \rightarrow \gamma \gamma$ and $A^{0} \rightarrow Z \gamma$ as a function of $M_{A^{0}}$ for various values of $\lambda_{4}$.

of $M_{A^{0}}$ in the low-mass region (130-300 GeV) and high-mass region (300-1000 GeV), respectively. The evolution of $\mathrm{BR}\left(A^{0} \rightarrow Z h\right)$ with $M_{A^{0}}$ and $\lambda_{4}$ is just opposite to that of $A^{0} \rightarrow b \bar{b}(t \bar{t})$ in the low- (high-) mass region. The variation of $\operatorname{BR}\left(A^{0} \rightarrow Z h\right)$ with $\lambda_{4}$ is dramatic below the $Z h$ threshold. In particular, near the $Z h$ threshold $\mathrm{BR}\left(A^{0} \rightarrow Z h\right) \sim 1.0$ for $\lambda_{4}=-1.0$, while $\mathrm{BR}\left(A^{0} \rightarrow Z h\right)$ tends to vanish for $\lambda_{4}=1.0$, which corresponds to the zero point of the $A^{0} Z h$ coupling:

$$
M_{\Delta}^{0}(Z h)=\sqrt{\left(\lambda_{4}+\lambda_{5}\right) v^{2}-M_{h}^{2}}
$$

with $\lambda_{4}+\lambda_{5}>2 M_{h}^{2} / v^{2} \approx 0.5 . \mathrm{BR}\left(A^{0} \rightarrow Z h\right)$ is totally dominant in the mass region between the $Z h$ and $t \bar{t}$ thresholds, and becomes comparable to $\mathrm{BR}\left(A^{0} \rightarrow t \bar{t}\right)$ when $M_{A^{0}}>2 M_{t}$.

At last, we study the one-loop-induced decays, $A^{0} \rightarrow \gamma \gamma, Z \gamma$. These two channels can only be induced by the top quark in the loop since the $A^{0} W^{+} W^{-}, A^{0} H^{+} H^{-}$, and $A^{0} H^{++} H^{--}$couplings are absent in the $C P$-conserving case. In Fig. 7, both $\mathrm{BR}\left(A^{0} \rightarrow \gamma \gamma\right)$ and $\mathrm{BR}\left(A^{0} \rightarrow Z \gamma\right)$ are displayed. For $M_{A^{0}}$ below the $Z h$ threshold, the variation in $\lambda_{4}$ of $\operatorname{BR}\left(A^{0} \rightarrow \gamma \gamma\right)$ increases as $M_{A^{0}}$ increases. $\operatorname{BR}\left(A^{0} \rightarrow \gamma \gamma\right)$ could reach $9 \times 10^{-4}$ for $M_{A^{0}} \approx 210 \mathrm{GeV}$ and $\lambda_{4}=1.0$, which is much smaller than the maximum of $\operatorname{BR}\left(H^{0} \rightarrow \gamma \gamma\right)$. The variation in $\lambda_{4}$ of $\mathrm{BR}\left(A^{0} \rightarrow Z \gamma\right)$ is slightly steeper, with a maximum of $1.2 \times 10^{-4}$ at $M_{A^{0}} \approx 215 \mathrm{GeV}$ and $\lambda_{4}=1.0$.

In the above, we have discussed the decay channels of $H^{0}$ and $A^{0}$ separately. We have shown that the scalar self-interactions have a large impact on their branching ratios. In Sec. IV, we will explore their LHC signatures. For this purpose, we choose the following benchmark values:

$$
v_{\Delta}=0.001 \mathrm{GeV}, \Delta M=5 \mathrm{GeV}, \lambda_{2}=\lambda_{3}=0.1, \lambda_{4}=0.25 .
$$



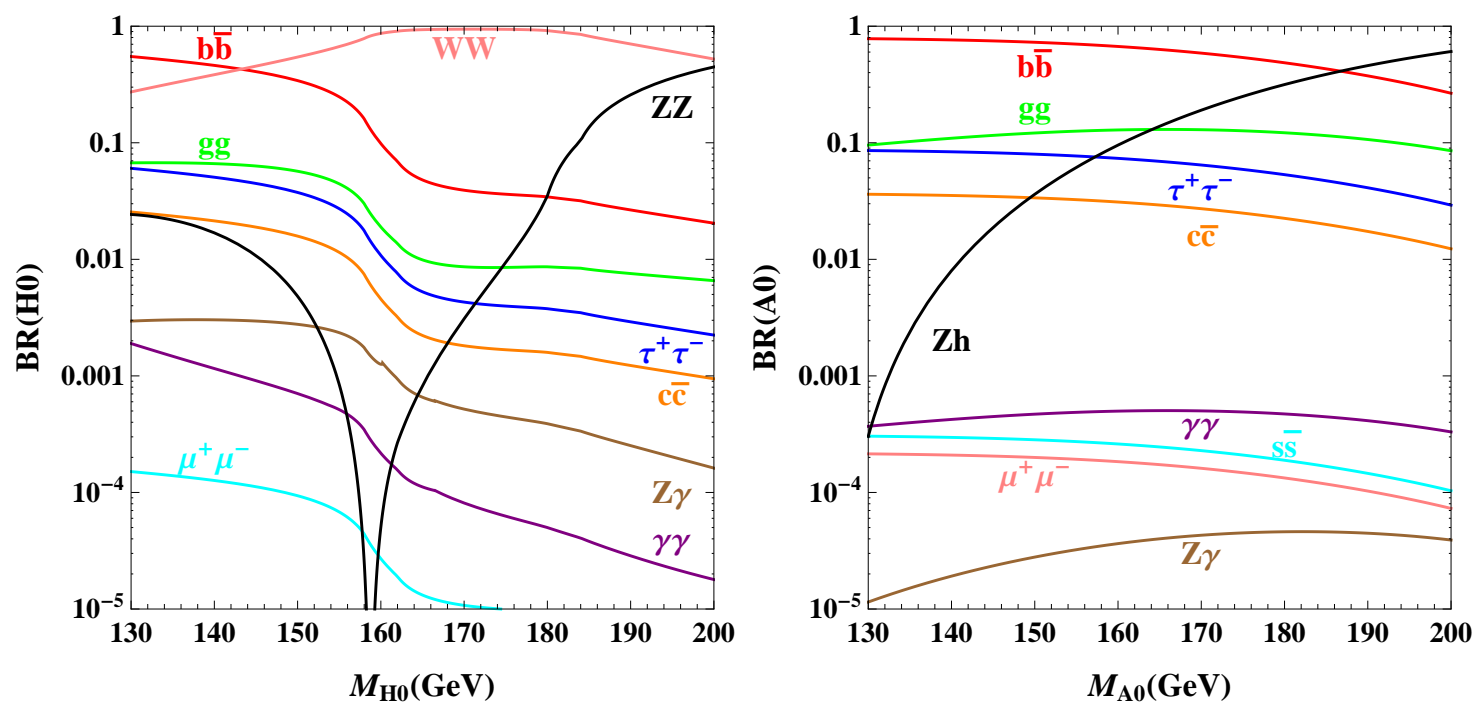

FIG. 8. Branching ratios of $H^{0} / A^{0}$ as a function of $M_{H^{0} / A^{0}}$ at the benchmark point in Eq. 99.

The reason that we set relatively small values of $v_{\Delta}$ and $\Delta M$ is to obtain large cascade decays of charged scalars as well as a large enhancement of neutral scalar production. In Fig. 8, we display all relevant branching ratios versus $M_{H^{0} / A^{0}}$ for this benchmark model, which is to be simulated in Sec. IV]for the LHC in the $b \bar{b} \gamma \gamma, b \bar{b} \tau^{+} \tau^{-}$, and $b \bar{b} W^{+} W^{-}$signal channels.

\section{PRODUCTION OF NEUTRAL HIGGS FROM CASCADE DECAYS}

We pointed out in Ref. [1] the importance of the associated $H^{0} A^{0}$ production in the nondegenerate case. To estimate the number of signal events, we simulated the signal channel $b \bar{b} \tau^{+} \tau^{-}$at $M_{H^{0} / A^{0}}=130 \mathrm{GeV}$. We found that, with a much higher production cross section than the SM Higgs pair $(h h)$ production, a $2.9 \sigma$ excess in that signal channel is achievable for LHC14@300. In the present work, we are interested in the observability of the associated $H^{0} A^{0}$ production in the nondecoupling mass regime $(130-200 \mathrm{GeV})$. In Fig. 9 we first show the production cross sections for a pair of various scalars at LHC14 versus $M_{\Delta}$ with a degenerate spectrum. As before, we incorporate the next-to-leading-order (NLO) QCD effects by multiplying a $K$-factor of 1.3 in all $q \bar{q}$ production channels [7]. The $h h$ production through gluon-gluon fusion at NLO (33 fb) is also indicated (black dashed line) for comparison. One can see that the cross section for $H^{0} A^{0}$ is about 20-500 $\mathrm{fb}$ in the mass region $130-300 \mathrm{GeV}$, which is much larger than the $h h$ production for most of the mass region and thus leads to great discovery potential.

In general, the new scalars are nondegenerate for a nonzero $\lambda_{5}$. In the positive scenario where $H^{ \pm \pm}$ are the lightest, the cascade decays of $H^{ \pm}$and $H^{0} / A^{0}$ can strengthen the observability of $H^{ \pm \pm}$[67, 68]. 

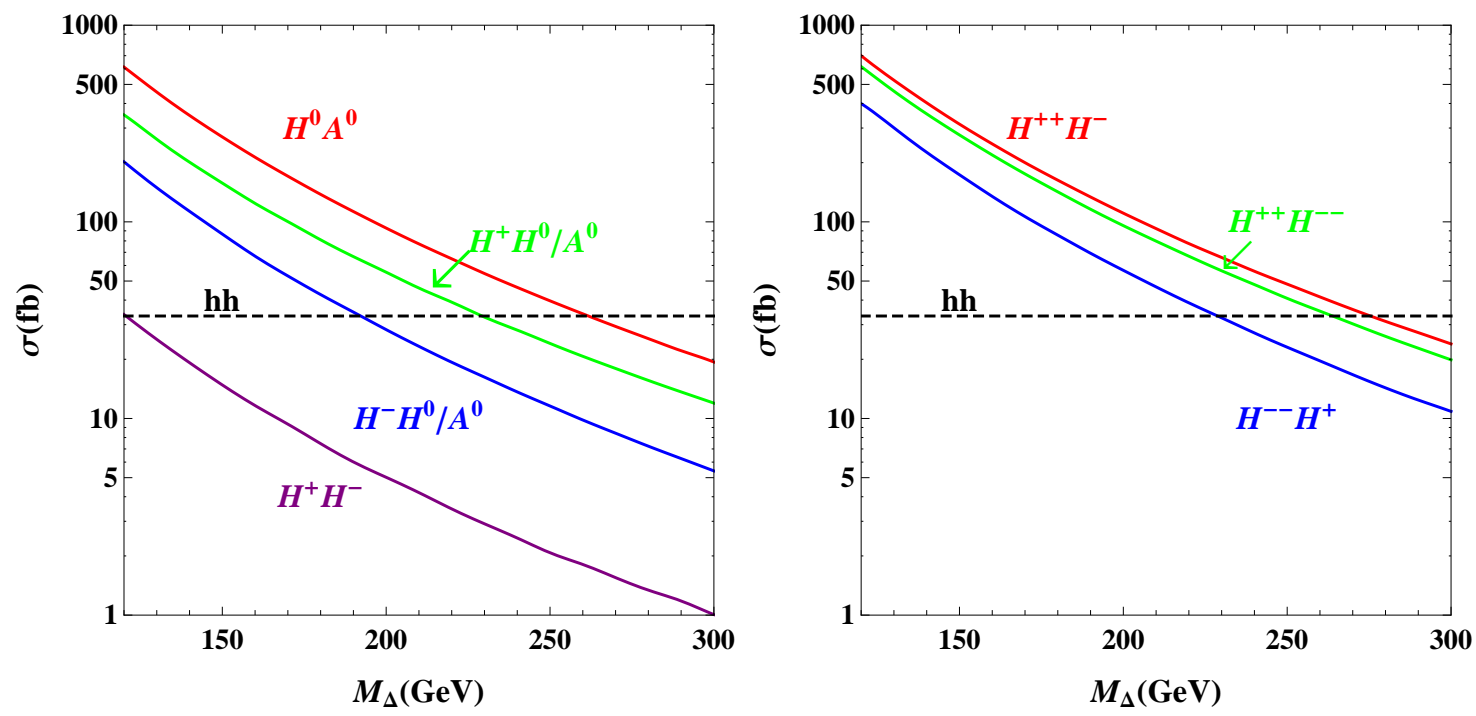

FIG. 9. Production cross sections for a pair of scalars at LHC14 versus $M_{\Delta}$ for a degenerate spectrum. The black dashed line is for the SM $h h$ production.

For the same reason, in the negative scenario where $H^{0} / A^{0}$ are the lightest, the charged scalars contribute instead to the production of $H^{0} / A^{0}$ through the cascade decays like $H^{ \pm} \rightarrow H^{0} / A^{0} W^{*}$. In this work, we study these contributions in the same way as was done for the positive scenario in Refs. [67, 68].

We define the reference cross section $X_{0}$ for the standard Drell-Yan process

$$
X_{0}=\sigma\left(p p \rightarrow Z^{*} \rightarrow H^{0} A^{0}\right)
$$

which is independent of the cascade decay parameters $v_{\Delta}$ and $\Delta M$. A detailed study on the $b \bar{b} \tau^{+} \tau^{-}$signal for this process with $M_{\Delta}=130 \mathrm{GeV}$ can be found in Ref. [1]. Besides the above direct production, neutral scalars can also be produced from cascade decays of charged scalars. These extra production channels include $H^{ \pm} H^{0} / A^{0}, H^{+} H^{-}, H^{ \pm} H^{\mp \mp}$, and $H^{++} H^{--}$followed by cascade decays of charged scalars. We consider first the associated $H^{ \pm} H^{0} / A^{0}$ production followed by cascade decays of $H^{ \pm}$,

$$
\begin{gathered}
p p \rightarrow W^{*} \rightarrow H^{ \pm} H^{0} \rightarrow H^{0} H^{0} W^{*}, \quad p p \rightarrow W^{*} \rightarrow H^{ \pm} H^{0} \rightarrow A^{0} H^{0} W^{*}, \\
p p \rightarrow W^{*} \rightarrow H^{ \pm} A^{0} \rightarrow H^{0} A^{0} W^{*}, \quad p p \rightarrow W^{*} \rightarrow H^{ \pm} A^{0} \rightarrow A^{0} A^{0} W^{*},
\end{gathered}
$$

resulting in three final states classified by a pair of neutral scalars: $A^{0} H^{0}, H^{0} H^{0}$, and $A^{0} A^{0}$. Noting that the last two originate only from cascade decays, any detection of such production channels would be a hint of charged scalars being involved. Using the fact that

$$
\begin{aligned}
\sigma\left(p p \rightarrow W^{*} \rightarrow H^{ \pm} H^{0}\right) & \simeq \sigma\left(p p \rightarrow W^{*} \rightarrow H^{ \pm} A^{0}\right), \\
\operatorname{BR}\left(H^{ \pm} \rightarrow H^{0} W^{*}\right) & \simeq \operatorname{BR}\left(H^{ \pm} \rightarrow A^{0} W^{*}\right),
\end{aligned}
$$


as well as the narrow width approximation, we calculate the production cross sections for these three final states:

$$
\begin{aligned}
& H^{0} A^{0}: X_{1}=2\left[\sigma\left(p p \rightarrow W^{+} \rightarrow H^{+} H^{0}\right)+\sigma\left(p p \rightarrow W^{-} \rightarrow H^{-} H^{0}\right)\right] \times \mathrm{BR}\left(H^{ \pm} \rightarrow A^{0} W^{*}\right), \\
& H^{0} H^{0}: Y_{1}=\left[\sigma\left(p p \rightarrow W^{+} \rightarrow H^{+} H^{0}\right)+\sigma\left(p p \rightarrow W^{-} \rightarrow H^{-} H^{0}\right)\right] \times \mathrm{BR}\left(H^{ \pm} \rightarrow H^{0} W^{*}\right), \\
& A^{0} A^{0}: Z_{1}=\left[\sigma\left(p p \rightarrow W^{+} \rightarrow H^{+} A^{0}\right)+\sigma\left(p p \rightarrow W^{-} \rightarrow H^{-} A^{0}\right)\right] \times \mathrm{BR}\left(H^{ \pm} \rightarrow A^{0} W^{*}\right) .
\end{aligned}
$$

The factor 2 in $X_{1}$ accounts for the equal contribution from the process with $H^{0}$ and $A^{0}$ interchanged. The relations $X_{1}=2 Y_{1}=2 Z_{1}$ actually hold true for all of the four production channels, since for a given channel the same branching ratios (such as for $H^{ \pm} \rightarrow H^{0} / A^{0} W^{*}$ ) are involved,

$$
X_{i}=2 Y_{i}=2 Z_{i},(i=1,2,3,4)
$$

where $X_{i}, Y_{i}$, and $Z_{i}$ refer to the cross sections for $H^{0} A^{0}, H^{0} H^{0}$, and $A^{0} A^{0}$ production with the subscript $i=1,2,3,4$ denoting the production channels $H^{ \pm} H^{0} / A^{0}, H^{+} H^{-}, H^{ \pm} H^{\mp \mp}$, and $H^{++} H^{--}$, respectively. The relations imply that we may concentrate on the cross section of $H^{0} A^{0}$ production.

Naively, one would expect the next important channel to be $\mathrm{H}^{+} \mathrm{H}^{-}$since it only involves two cascade decays:

$$
X_{2}=2 \sigma\left(p p \rightarrow \gamma^{*} / Z^{*} \rightarrow H^{+} H^{-}\right) \times \mathrm{BR}\left(H^{ \pm} \rightarrow H^{0} W^{*}\right) \mathrm{BR}\left(H^{ \pm} \rightarrow A^{0} W^{*}\right) .
$$

But as already mentioned in Ref. [68], a smaller coupling and destructive interference between the $\gamma^{*}$ and $Z^{*}$ exchange make the cross section of $H^{+} H^{-}$production an order of magnitude smaller than that of $H^{0} A^{0}$ even for a degenerate spectrum. Considering further suppression due to cascade decays, $X_{2}$ is not important for the enhancement of $H^{0} A^{0}$ production and can be safely neglected in the numerical analysis.

The contribution from $H^{ \pm} H^{\mp \mp}$ is more important despite the fact that it involves three cascade decays:

$$
\begin{aligned}
X_{3}= & 2\left[\sigma\left(p p \rightarrow W^{-*} \rightarrow H^{+} H^{--}\right)+\sigma\left(p p \rightarrow W^{+*} \rightarrow H^{-} H^{++}\right)\right] \times \\
& \operatorname{BR}\left(H^{ \pm \pm} \rightarrow H^{ \pm} W^{*}\right) \operatorname{BR}\left(H^{ \pm} \rightarrow H^{0} W^{*}\right) \operatorname{BR}\left(H^{ \pm} \rightarrow A^{0} W^{*}\right) .
\end{aligned}
$$

As shown in Fig. 9, $\sigma\left(p p \rightarrow W^{*} \rightarrow H^{ \pm} H^{\mp \mp}\right)$ is the largest for a degenerate mass spectrum. When cascade decays are dominant, the phase-space suppression of heavy charged scalars will be important. So we expect that the $H^{0} A^{0}$ production receives considerable enhancement from $H^{ \pm} H^{\mp \mp}$ when the mass splitting is small and cascade decays are dominant.

Finally, the last mechanism is $H^{++} H^{--}$, which involves four cascade decays:

$$
\begin{aligned}
X_{4}= & 2 \sigma\left(p p \rightarrow \gamma^{*} / Z^{*} \rightarrow H^{++} H^{--}\right) \times \mathrm{BR}\left(H^{ \pm \pm} \rightarrow H^{ \pm} W^{*}\right)^{2} \\
& \times \mathrm{BR}\left(H^{ \pm} \rightarrow H^{0} W^{*}\right) \mathrm{BR}\left(H^{ \pm} \rightarrow A^{0} W^{*}\right) .
\end{aligned}
$$



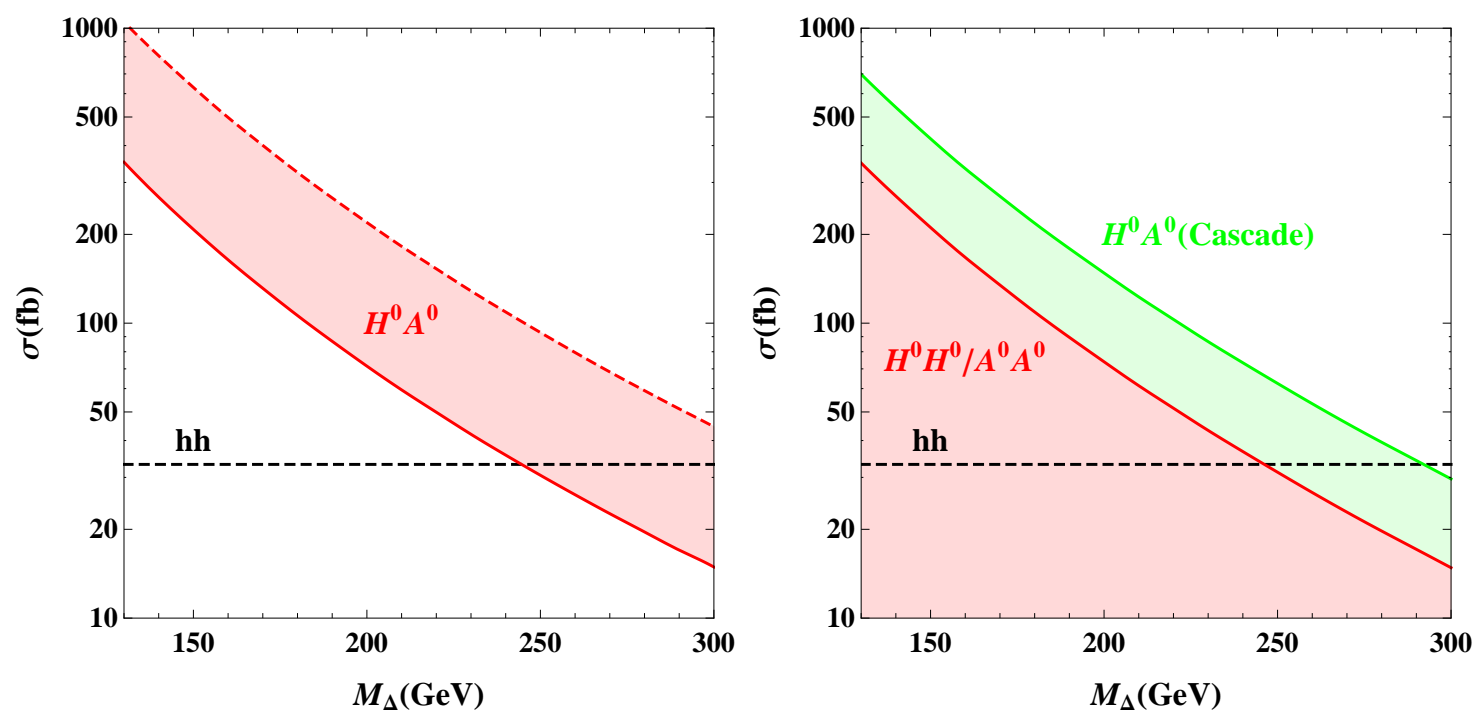

FIG. 10. Production cross sections for a pair of neutral scalars versus $M_{\Delta}$ at LHC14 and with $\Delta M=5 \mathrm{GeV}$, $v_{\Delta}=0.001 \mathrm{GeV}$. Left: The red solid (dashed) line corresponds to $X_{0}(X)$. Right: The red line corresponds to $H^{0} H^{0} / A^{0} A^{0}$ from cascade decays $Y / Z$, and the green line to $H^{0} A^{0}$ from cascade decays $\left(X_{C}\right)$. The shaded regions are filled by scanning over $\Delta M$ and $v_{\Delta}$.

This mechanism is also promising since the cross section of $H^{++} H^{--}$production is slightly larger than $H^{0} A^{0}$ production for a degenerate mass spectrum. The phase-space suppression of $X_{4}$ is more severe than that of $X_{3}$, because a pair of the heaviest $H^{ \pm \pm}$are produced.

Summing over all four of the above channels yields the contribution to the $H^{0} A^{0}$ production from cascade decays,

$$
X_{C}=X_{1}+X_{2}+X_{3}+X_{4}
$$

and the total production cross section of $H^{0} A^{0}$ is then $X=X_{0}+X_{C}$. Using Eq. (17), the total cross sections for the pair production $H^{0} H^{0} / A^{0} A^{0}, Y=\sum_{i} Y_{i}, Z=\sum_{i} Z_{i}$, are given by

$$
Y=Z=\frac{1}{2} X_{C}
$$

Since the enhancement from cascade decays depends on a not severely suppressed phase space and a larger branching ratio of cascade decays, we choose to work with a relatively smaller mass splitting and triplet vev as shown in Eq. (9). Figure 10 displays the cross sections of the $H^{0} A^{0}, H^{0} H^{0}$, and $A^{0} A^{0}$ production as a function of $M_{\Delta}$. As can be seen from the figure, the production of $H^{0} A^{0}$ can be enhanced by a factor of 3 , while the $H^{0} H^{0} / A^{0} A^{0}$ production at the maximal enhancement can reach the level of $X_{0}$. This could make the detection of neutral scalar pair productions very promising in the negative scenario. 


\section{LHC SIGNATURES OF NEUTRAL SCALAR PRODUCTION}

In this section we investigate the signatures of neutral scalar production at the LHC. From previous studies on the SM $h h$ production, we already know that the most promising signal is $b \bar{b} \gamma \gamma$, and $b \bar{b} \tau^{+} \tau^{-}$is next to it, while both semileptonic and dileptonic decays of $W^{\prime}$ 's in the $b \bar{b} W^{+} W^{-}$channel are challenging. In this work we analyze all three of the signals $-b \bar{b} \gamma \gamma, b \bar{b} \tau^{+} \tau^{-}$, and $b \bar{b} W^{+} W^{-} \rightarrow b \bar{b} \ell^{+} \ell^{-} 2 \nu(\ell=e, \mu$ for collider identification) — as well as their backgrounds based on the benchmark model presented in Eq. 99.

In Sec. III we discussed the Drell-Yan production of $H^{0} A^{0}$ and the enhanced pair and associated production of neutral scalars $H^{0} / A^{0}$ due to cascade decays of charged scalars $H^{ \pm}, H^{ \pm \pm}$. We are now ready to incorporate the branching ratios of $H^{0} / A^{0}$ decays for a specific signal channel. For instance, the cross sections for the $b \bar{b} \gamma \gamma$ signal channel can be written as

$$
\begin{aligned}
S_{0}(b \bar{b} \gamma \gamma)= & X_{0} \times\left[\mathrm{BR}\left(H^{0} \rightarrow b \bar{b}\right) \mathrm{BR}\left(A^{0} \rightarrow \gamma \gamma\right)+\operatorname{BR}\left(H^{0} \rightarrow \gamma \gamma\right) \operatorname{BR}\left(A^{0} \rightarrow b \bar{b}\right)\right], \\
S(b \bar{b} \gamma \gamma)= & X \times\left[\operatorname{BR}\left(H^{0} \rightarrow b \bar{b}\right) \operatorname{BR}\left(A^{0} \rightarrow \gamma \gamma\right)+\operatorname{BR}\left(H^{0} \rightarrow \gamma \gamma\right) \operatorname{BR}\left(A^{0} \rightarrow b \bar{b}\right)\right] \\
& +2 Y \times \operatorname{BR}\left(H^{0} \rightarrow b \bar{b}\right) \operatorname{BR}\left(H^{0} \rightarrow \gamma \gamma\right)+2 Z \times \operatorname{BR}\left(A^{0} \rightarrow b \bar{b}\right) \operatorname{BR}\left(A^{0} \rightarrow \gamma \gamma\right)
\end{aligned}
$$

Here $S_{0}$ denotes the signal from the direct production $p p \rightarrow Z^{*} \rightarrow H^{0} A^{0}$ alone, and $S$ includes contributions from cascade decays. $S_{(0)}\left(b \bar{b} \tau^{+} \tau^{-}\right)$has a similar expression as $S_{(0)}(b \bar{b} \gamma \gamma)$, while $S_{(0)}\left(b \bar{b} \ell^{+} \ell^{-} 2 \nu\right)$ is simpler since the decay mode $A^{0} \rightarrow W^{+} W^{-}$is absent.

The theoretical cross sections for the $b \bar{b} \gamma \gamma, b \bar{b} \tau^{+} \tau^{-}$, and $b \bar{b} \ell^{+} \ell^{-} 2 \nu$ signal channels are plotted in Fig. 11. The cross section $S_{0}(b \bar{b} \gamma \gamma) / S_{0}\left(b \bar{b} \tau^{+} \tau^{-}\right)$is larger than that of the SM $h h$ production until $M_{\Delta}=159 / 161 \mathrm{GeV}$; taking into account cascade enhancement pushes the corresponding $M_{\Delta}$ further to $179 / 197 \mathrm{GeV} . S_{0}\left(b \bar{b} \ell^{+} \ell^{-} 2 \nu\right)$ is always larger than that of $h h$ in the mass region $130-200 \mathrm{GeV}$, and interestingly, it keeps about the same value when $M_{\Delta}<160 \mathrm{GeV}$. The signal from $H^{0} H^{0}$ is comparable with $S_{0}$ in these three channels only for $M_{\Delta}<160 \mathrm{GeV}$, while in contrast the signal from $A^{0} A^{0}$ becomes dominant for the $b \bar{b} \gamma \gamma$ and $b \bar{b} \tau^{+} \tau^{-}$channels when $M_{\Delta}>160 \mathrm{GeV}$. Therefore, we have a chance to probe the $A^{0} A^{0}$ pair production in these two channels. Also shown in Fig. 11 is the enhancement factor $S / S_{0}$ for the three signal channels at the benchmark point (9) as a function of $M_{\Delta}$, which will help us understand the simulation results.

\section{A. $b \bar{b} \gamma \gamma$ signal channel}

In our simulation, the parton-level signal and background events are generated with MADGRAPH5 [69]. We perform parton shower and fast detector simulations with PYTHIA [70] and DELPHES3 [71]. Finally, 

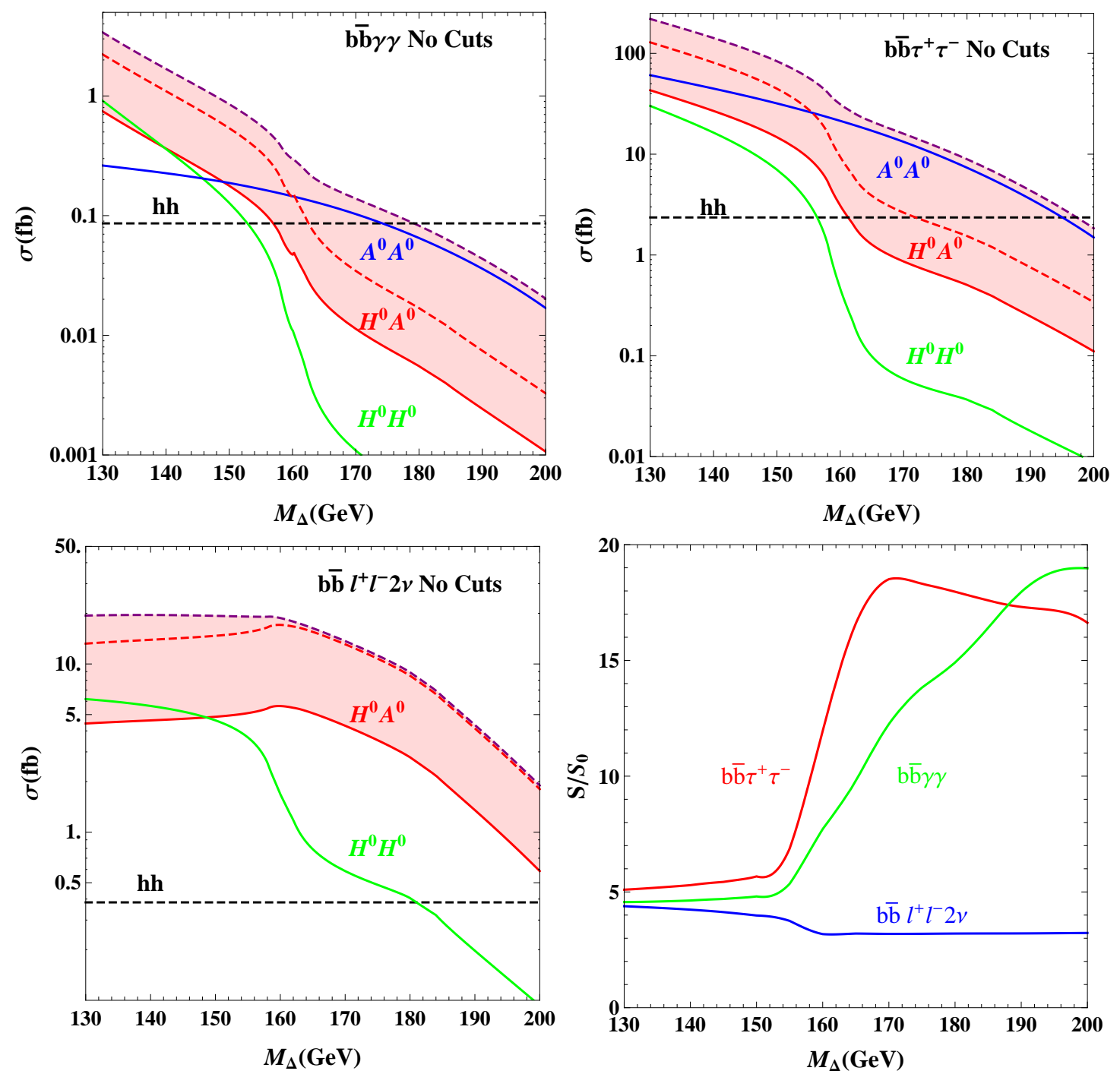

FIG. 11. Theoretical cross sections of $b \bar{b} \gamma \gamma, b \bar{b} \tau^{+} \tau^{-}$, and $b \bar{b} \ell^{+} \ell^{-} 2 \nu$ signal channels at LHC14. The red solid (dashed) line corresponds to the signal from $X_{0}(X)$, the green (blue) solid line corresponds to the signal from $Y(Z)$, and the purple dashed line shows the total cross section $S$ for the signal. The SM $h h$ cross section is shown for comparison. The lower right panel shows the enhancement factor $S / S_{0}$ in the three signal channels.

MADANALYSIS5 [72] is responsible for data analysis and plotting. We take a flat $b$-tagging efficiency of 70\%, and mistagging rates of $10 \%$ for $c$ jets and $1 \%$ for light-flavor jets, respectively. Jet reconstruction is done using the anti- $k_{T}$ algorithm with a radius parameter of $R=0.5$. We further assume a photon identification efficiency of $85 \%$ and a jet-faking-photon rate of $1.2 \times 10^{-4}$ [73]. 
The main SM backgrounds to the signal are as follows:

$$
\begin{aligned}
& b \bar{b} \gamma \gamma: p p \rightarrow b \bar{b} \gamma \gamma \\
& t \bar{t} h: p p \rightarrow t \bar{t} h \rightarrow b \ell^{+} \nu \bar{b} \ell^{-} \nu \gamma \gamma\left(\ell^{ \pm} \text {missed }\right) \\
& Z h: p p \rightarrow Z h \rightarrow b \bar{b} \gamma \gamma
\end{aligned}
$$

Among them, $b \bar{b} \gamma \gamma$ and $Z h$ are irreducible, while $t \bar{t} h$ is reducible and can be reduced by vetoing the additional $\ell$ 's with $p_{T}^{\ell}>20 \mathrm{GeV}$ and $\left|\eta_{\ell}\right|<2.4$. In addition, there exist many reducible sources of fake $b \bar{b} \gamma \gamma:$

$$
\begin{gathered}
p p \rightarrow b \bar{b} j j \nrightarrow b \bar{b} \gamma \gamma, p p \rightarrow b \bar{b} j \gamma \nrightarrow b \bar{b} \gamma \gamma, \ldots \\
p p \rightarrow c \bar{c} \gamma \gamma \nrightarrow b \bar{b} \gamma \gamma, p p \rightarrow j \bar{j} \gamma \gamma \nrightarrow b \bar{b} \gamma \gamma, \ldots,
\end{gathered}
$$

where $x \nrightarrow y$ stands for a final-state $x$ misidentified as $y$. The remaining fake sources are subdominant and are thus not included in our simulation. The QCD corrections to the backgrounds are included by a multiplicative $K$-factor of 1.10 and 1.33 for the leading cross sections of $t \bar{t} h$ and $Z h$ at LHC14 [74], respectively. The cross section of the $b \bar{b} \gamma \gamma$ background has been normalized to include fake sources and does not take NLO corrections into account.

The distributions of some kinematical variables before applying any cuts are shown in Fig. 12, where we assume $M_{\Delta}=130,160,190 \mathrm{GeV}$. In our analysis, we require that the final states include exactly one $b$-jet pair and one $\gamma$ pair and satisfy the following basic cuts:

$$
p_{T}^{b, \gamma}>30 \mathrm{GeV},\left|\eta_{b, \gamma}\right|<2.4, \Delta R_{b b, \gamma \gamma, b \gamma}>0.4
$$

where $\Delta R=\sqrt{(\Delta \phi)^{2}+(\Delta \eta)^{2}}$ is the particle separation, with $\Delta \phi$ and $\Delta \eta$ being the separation in the azimuthal angle and rapidity, respectively. Here we employ a tighter $p_{T}$ cut than is usually applied to suppress the QCD-electroweak $b \bar{b} \gamma \gamma$ background. The $b$-jet pair and $\gamma$ pair are then required to fall in the following windows on the invariant masses and fulfill the $\Delta R$ cut criteria:

$$
\begin{gathered}
\Delta R_{b b}<2.5,\left|M_{b b}-M_{\Delta}\right|<15 \mathrm{GeV} \\
\Delta R_{\gamma \gamma}<2.5,\left|M_{\gamma \gamma}-M_{\Delta}\right|<10 \mathrm{GeV} .
\end{gathered}
$$

As shown in Fig. 12, the $\Delta R_{b b, \gamma \gamma}$ distributions of the signal are clearly more compact as they are more likely coming from the same particles. Thus the $\Delta R$ cuts can effectively suppress the background. More specific cuts are necessary for further analysis. A useful variable is the invariant mass of the neutral scalar pair $M_{H^{0} A^{0}}$, and the total transverse energy $E_{T}$ is also distinctive. The peak of $M_{H^{0} A^{0}}$ increases with $M_{\Delta}$, and similarly for $E_{T}$. For simplicity, we adopt for the cuts a linear shift between $M_{H^{0} A^{0}}, E_{T}$ and $M_{\Delta}$ :

$$
M_{H^{0} A^{0}}>2 M_{\Delta}+90 \mathrm{GeV}, E_{T}>2 M_{\Delta}-60 \mathrm{GeV} .
$$



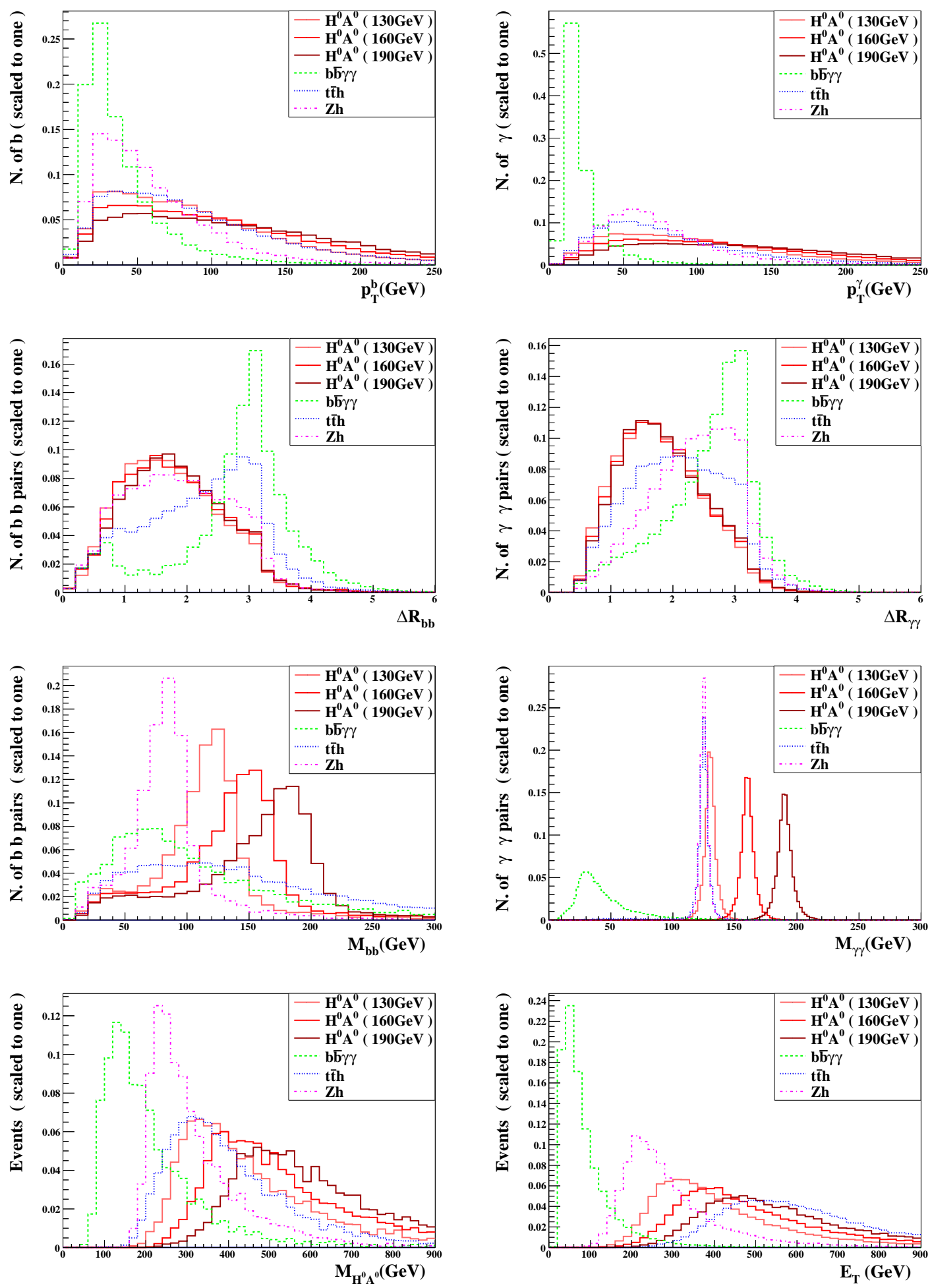

FIG. 12. Distributions of $p_{T}^{b, \gamma}, \Delta R_{b b, \gamma \gamma}, M_{b b, \gamma \gamma, H^{0} A^{0}}$, and $E_{T}$ for the signal $b \bar{b} \gamma \gamma$ and its backgrounds before applying any cuts at LHC14. 


\begin{tabular}{|c|c|c|c|c|c|c|}
\hline$M_{\Delta}=130 \mathrm{GeV}$ & $H^{0} A^{0}\left(S_{0}\right)$ & $b \bar{b} \gamma \gamma$ & $t \bar{t} h$ & $Z h$ & $S / B$ & $\mathcal{S}(S, B)$ \\
\hline Cross section at NLO & $8.01 \times 10^{-1}$ & $5.92 \times 10^{3}$ & 1.18 & $2.99 \times 10^{-1}$ & $1.39 \times 10^{-4}$ & $5.75 \times 10^{-1}$ \\
\hline Basic cuts & $1.22 \times 10^{-1}$ & $4.16 \times 10^{1}$ & $1.03 \times 10^{-1}$ & $3.41 \times 10^{-2}$ & $2.92 \times 10^{-3}$ & 1.03 \\
\hline Reconstruct scalars from $b \mathrm{~s}$ & $6.99 \times 10^{-2}$ & 7.07 & $1.50 \times 10^{-2}$ & $9.61 \times 10^{-4}$ & $9.87 \times 10^{-3}$ & 1.44 \\
\hline Reconstruct scalars from $\gamma \mathrm{s}$ & $5.28 \times 10^{-2}$ & $1.03 \times 10^{-1}$ & $1.08 \times 10^{-2}$ & $7.32 \times 10^{-4}$ & $4.63 \times 10^{-1}$ & 8.01 \\
\hline Cut on $M_{H^{0} A^{0}}$ & $4.21 \times 10^{-2}$ & $2.04 \times 10^{-2}$ & $4.69 \times 10^{-3}$ & $3.23 \times 10^{-4}$ & 1.65 & 12.0 \\
\hline Cut on $E_{T}$ & $3.31 \times 10^{-2}$ & $6.58 \times 10^{-3}$ & $4.68 \times 10^{-3}$ & $2.27 \times 10^{-4}$ & 2.88 & 12.8 \\
\hline Cascade enhanced & $1.51 \times 10^{-1}$ & - & - & - & 13.1 & 41.0 \\
\hline$M_{\Delta}=160 \mathrm{GeV}$ & $H^{0} A^{0}\left(S_{0}\right)$ & $b \bar{b} \gamma \gamma$ & $t \bar{t} h$ & $Z h$ & $S / B$ & $\mathcal{S}(S, B)$ \\
\hline Cross section at NLO & $5.10 \times 10^{-2}$ & $5.92 \times 10^{3}$ & 1.18 & $2.99 \times 10^{-1}$ & $8.61 \times 10^{-6}$ & $3.63 \times 10^{-2}$ \\
\hline Basic cuts & $8.78 \times 10^{-3}$ & $4.16 \times 10^{1}$ & $1.03 \times 10^{-1}$ & $3.41 \times 10^{-2}$ & $2.10 \times 10^{-4}$ & $7.44 \times 10^{-2}$ \\
\hline Reconstruct scalars from $b \mathrm{~s}$ & $4.11 \times 10^{-3}$ & 5.06 & $1.34 \times 10^{-2}$ & $2.36 \times 10^{-4}$ & $8.11 \times 10^{-4}$ & $9.99 \times 10^{-2}$ \\
\hline Reconstruct scalars from $\gamma \mathrm{s}$ & $3.27 \times 10^{-3}$ & $3.42 \times 10^{-2}$ & $1.57 \times 10^{-5}$ & 0.00 & $9.56 \times 10^{-2}$ & $9.53 \times 10^{-1}$ \\
\hline Cut on $M_{H^{0} A^{0}}$ & $2.57 \times 10^{-3}$ & $1.12 \times 10^{-2}$ & $1.18 \times 10^{-5}$ & 0.00 & $2.30 \times 10^{-1}$ & 1.28 \\
\hline Cut on $E_{T}$ & $1.73 \times 10^{-3}$ & $3.95 \times 10^{-3}$ & $1.03 \times 10^{-5}$ & 0.00 & $4.37 \times 10^{-1}$ & 1.41 \\
\hline Cascade enhanced & $1.10 \times 10^{-2}$ & - & - & - & 2.77 & 7.29 \\
\hline$M_{\Delta}=190 \mathrm{GeV}$ & $H^{0} A^{0}\left(S_{0}\right)$ & $b \bar{b} \gamma \gamma$ & $t \bar{t} h$ & $Z h$ & $S / B$ & $\mathcal{S}(S, B)$ \\
\hline Cross section at NLO & $2.68 \times 10^{-3}$ & $5.92 \times 10^{3}$ & 1.18 & $2.99 \times 10^{-1}$ & $4.53 \times 10^{-7}$ & $1.91 \times 10^{-3}$ \\
\hline Basic cuts & $5.33 \times 10^{-4}$ & $4.16 \times 10^{1}$ & $1.03 \times 10^{-1}$ & $3.41 \times 10^{-2}$ & $1.28 \times 10^{-5}$ & $4.52 \times 10^{-3}$ \\
\hline Reconstruct scalars from $b$ s & $2.27 \times 10^{-4}$ & 3.61 & $1.05 \times 10^{-2}$ & $1.24 \times 10^{-4}$ & $6.27 \times 10^{-5}$ & $6.53 \times 10^{-3}$ \\
\hline Reconstruct scalars from $\gamma \mathrm{s}$ & $1.81 \times 10^{-4}$ & $2.47 \times 10^{-2}$ & $3.93 \times 10^{-6}$ & 0.00 & $7.34 \times 10^{-3}$ & $6.30 \times 10^{-2}$ \\
\hline Cut on $M_{H^{0} A^{0}}$ & $1.55 \times 10^{-4}$ & $9.87 \times 10^{-3}$ & $3.93 \times 10^{-6}$ & 0.00 & $1.57 \times 10^{-2}$ & $8.52 \times 10^{-2}$ \\
\hline Cut on $E_{T}$ & $8.35 \times 10^{-5}$ & $1.48 \times 10^{-3}$ & $3.93 \times 10^{-6}$ & 0.00 & $5.63 \times 10^{-2}$ & $1.18 \times 10^{-1}$ \\
\hline Cascade enhanced & $1.50 \times 10^{-3}$ & - & - & - & 1.01 & 1.87 \\
\hline
\end{tabular}

TABLE I. Evolution of signal and background cross sections (in fb) at LHC14 for the $b \bar{b} \gamma \gamma$ signal channel upon imposing the cuts one by one. For the cascade-enhanced signal only the cross section passing all the cuts is shown. The last two columns assume an integrated luminosity of $3000 \mathrm{fb}^{-1}$.

For instance, we apply $M_{H^{0} A^{0}}>350 \mathrm{GeV}, E_{T}>200 \mathrm{GeV}$ at the benchmark point $M_{\Delta}=130 \mathrm{GeV}$.

To estimate the observability quantitatively, we adopt the following significance measurement:

$$
\mathcal{S}(S, B)=\sqrt{2\left((S \cdot \mathcal{L}+B \cdot \mathcal{L}) \log \left(1+\frac{S}{B}\right)-S \cdot \mathcal{L}\right)},
$$

which is more suitable than the usual definition of $S / \sqrt{B}$ or $S / \sqrt{S+B}$ for Monte Carlo analysis [75]. Here $S$ and $B$ are the signal and background cross sections, and $\mathcal{L}$ is the integrated luminosity. The survival cross sections of the signal from the Drell-Yan process and of the backgrounds upon imposing cuts step by 
step are summarized in Table I at the benchmark point (9) for $M_{\Delta}=130,160,190 \mathrm{GeV}$ respectively. For the cascade-enhanced signal, only the cross section passing all the cuts is shown. The last two columns in the table show the signal-to-background ratio $S / B$ and the statistical significance $\mathcal{S}(S, B)$.

For $M_{\Delta}=130 \mathrm{GeV}$, the $b \bar{b} \gamma \gamma$ channel is very promising. Without (with) cascade enhancement, the final significance can reach 12.8 (41) for LHC14@3000, corresponding to 99 (453) events. For $M_{\Delta}=160 \mathrm{GeV}$, the channel becomes challenging since the cross section has decreased by a factor of 15.7 compared with the case of $M_{\Delta}=130 \mathrm{GeV}$. But the cuts we applied are efficient to suppress the SM background, and with cascade enhancement the significance could still reach 7.29 for $3000 \mathrm{fb}^{-1}$, corresponding to 33 events in the most optimistic case. For $M_{\Delta}=190 \mathrm{GeV}$, it looks hopeless even with maximal cascade enhancement in our benchmark model: to achieve 10 signal events, an integrated luminosity of at least $6670 \mathrm{fb}^{-1}$ is required, which is beyond the reach of the future LHC.

\section{B. $b \bar{b} \tau^{+} \tau^{-}$signal channel}

For this signal channel, an important part of the analysis depends on the ability to reconstruct the $b$ pair and the $\tau$ pair. Here we consider the hadronic decays of the $\tau$ lepton and assume a $\tau$-tagging efficiency of $70 \%$ with a negligible fake rate.

The main SM backgrounds are as follows:

$$
\begin{gathered}
b \bar{b} \tau^{+} \tau^{-}: p p \rightarrow b \bar{b} Z / \gamma^{*} / h \rightarrow b \bar{b} \tau^{+} \tau^{-}, \\
b \bar{b} W^{+} W^{-}: p p \rightarrow b \bar{b} W^{+} W^{-} \rightarrow b \bar{b} \tau^{+} \nu_{\tau} \tau^{-} \bar{\nu}_{\tau}, \\
Z h: p p \rightarrow Z h \rightarrow b \bar{b} \tau^{+} \tau^{-} .
\end{gathered}
$$

The irreducible QCD-electroweak background comes from $b \bar{b} \tau^{+} \tau^{-}$, where the $\tau$ pair originates from the decays of $Z / \gamma^{*} / h$. Since the hadronic decays of $\tau$ always contain neutrinos, we also include the SM background $b \bar{b} W^{+} W^{-}$, which contributes to the $b \bar{b} \tau^{+} \nu_{\tau} \tau^{-} \bar{\nu}_{\tau}$ final state. The $b \bar{b} W^{+} W^{-}$background mainly originates from $t \bar{t}$ production with subsequent decays $t \rightarrow b W$ and $W \rightarrow \tau \nu_{\tau}$. Moreover, the associated $Z h$ production gets involved through the subsequent decays $h \rightarrow b \bar{b}$ and $Z \rightarrow \tau^{+} \tau^{-}$or vice versa. The QCD corrections to the backgrounds are included by a multiplicative $K$-factor of $1.21,1.35$, and 1.33 to the leading cross section of $b \bar{b} \tau^{+} \tau^{-}[76], t \bar{t}$ [77], and $Z h$ [74] at LHC14.

The kinematical distributions similar to the $b \bar{b} \gamma \gamma$ channel are shown in Fig. 13 . As one can see from the figure, the $\tau$ jets are less energetic than the $b$ jets (similar to those in the $b \bar{b} \gamma \gamma$ signal channel) due to missing neutrinos in the final state. We first employ the following selection cuts to pick up signals with exactly one 

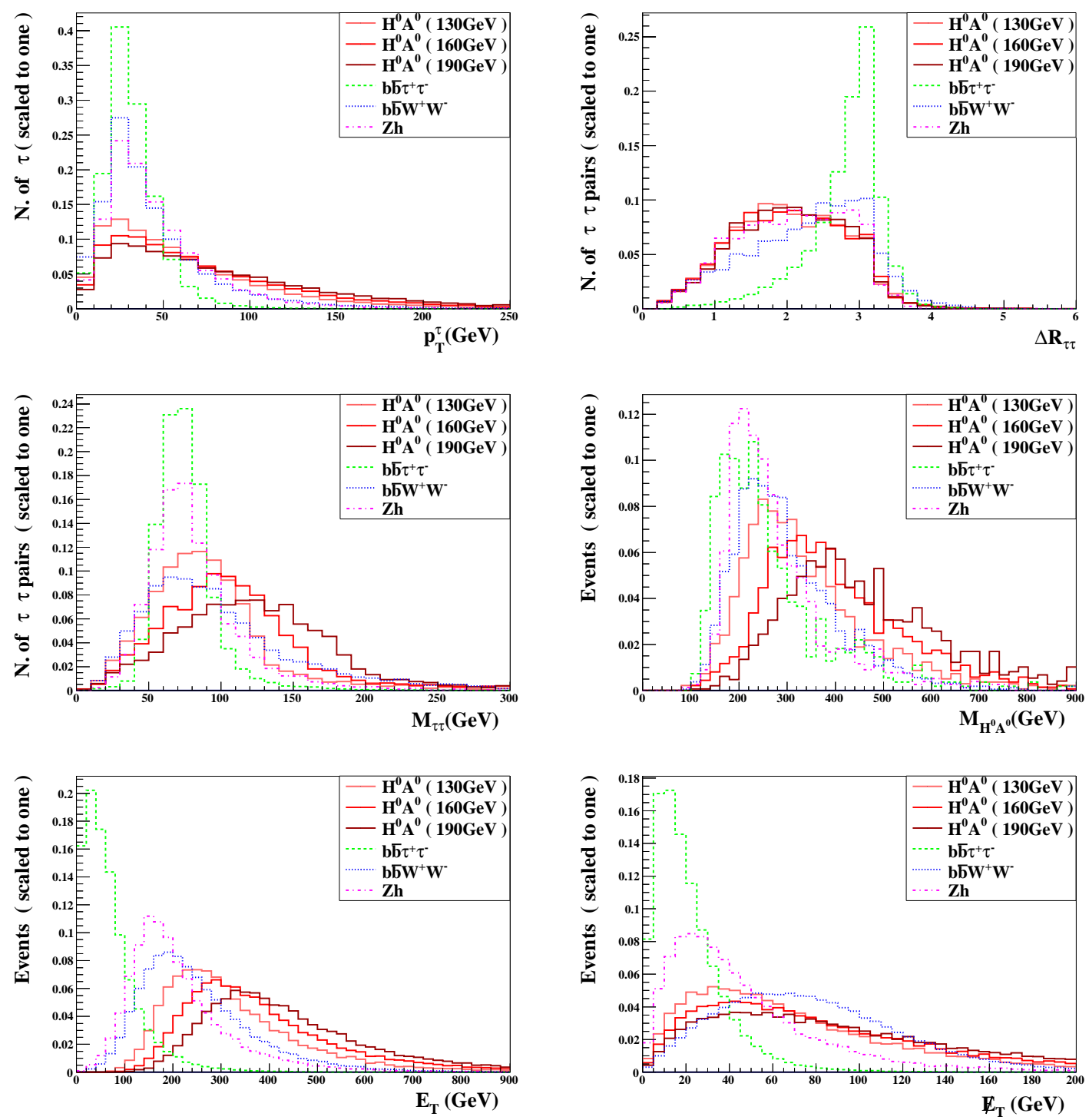

FIG. 13. Distributions of $p_{T}^{\tau}, \Delta R_{\tau \tau}, M_{\tau \tau, H^{0} A^{0}}, E_{T}$, and $E_{T}$ for the signal $b \bar{b} \tau^{+} \tau^{-}$and its backgrounds before applying any cuts at LHC14.

$b$ pair and one $\tau$ pair:

$$
p_{T}^{b, \tau}>30 \mathrm{GeV},\left|\eta_{b, \tau}\right|<2.4, \Delta R_{b b, b \tau, \tau \tau}>0.4
$$

and no cut on $E_{T}$ is adopted. After the selection, the $\tau$ and $b$ pairs are required to fulfill the cuts on the invariant masses and separations:

$$
\begin{gathered}
\Delta R_{\tau \tau}<2.5, M_{\Delta}-40 \mathrm{GeV}<M_{\tau \tau}<M_{\Delta}, \\
\Delta R_{b b}<2.5,\left|M_{b b}-M_{\Delta}\right|<15 \mathrm{GeV} .
\end{gathered}
$$




\begin{tabular}{|c|c|c|c|c|c|c|}
\hline$M_{\Delta}=130 \mathrm{GeV}$ & $H^{0} A^{0}\left(S_{0}\right)$ & $b \bar{b} \tau^{+} \tau^{-}$ & $b \bar{b} W^{+} W^{-}$ & $Z h$ & $S / B$ & $\mathcal{S}(S, B)$ \\
\hline Cross section at NLO & $4.31 \times 10^{1}$ & $3.10 \times 10^{4}$ & $7.92 \times 10^{3}$ & $2.21 \times 10^{1}$ & $1.11 \times 10^{-3}$ & 12.0 \\
\hline Basic cuts & $7.75 \times 10^{-1}$ & $4.49 \times 10^{1}$ & $8.97 \times 10^{1}$ & $2.91 \times 10^{-1}$ & $5.74 \times 10^{-3}$ & 3.65 \\
\hline Reconstruct scalars from $\tau \mathrm{s}$ & $5.14 \times 10^{-1}$ & $1.19 \times 10^{1}$ & $3.57 \times 10^{1}$ & $1.06 \times 10^{-1}$ & $1.08 \times 10^{-2}$ & 4.06 \\
\hline Reconstruct scalars from $b \mathrm{~s}$ & $2.14 \times 10^{-1}$ & 4.34 & $9.44 \times 10^{-1}$ & $2.28 \times 10^{-2}$ & $4.04 \times 10^{-2}$ & 5.06 \\
\hline Cut on $M_{H^{0} A^{0}}$ & $1.29 \times 10^{-1}$ & $1.96 \times 10^{-1}$ & $1.51 \times 10^{-1}$ & $8.10 \times 10^{-3}$ & $3.64 \times 10^{-1}$ & 11.3 \\
\hline Cut on $E_{T}$ & $1.03 \times 10^{-1}$ & $9.87 \times 10^{-2}$ & $7.35 \times 10^{-2}$ & $5.89 \times 10^{-3}$ & $5.81 \times 10^{-1}$ & 12.4 \\
\hline Cascade enhanced & $5.27 \times 10^{-1}$ & - & - & - & 2.96 & 51.6 \\
\hline$M_{\Delta}=160 \mathrm{GeV}$ & $H^{0} A^{0}\left(S_{0}\right)$ & $b \bar{b} \tau^{+} \tau^{-}$ & $b \bar{b} W^{+} W^{-}$ & $Z h$ & $S / B$ & $\mathcal{S}(S, B)$ \\
\hline Cross section at NLO & 3.08 & $3.10 \times 10^{4}$ & $7.92 \times 10^{3}$ & $2.21 \times 10^{1}$ & $7.92 \times 10^{-5}$ & $8.55 \times 10^{-1}$ \\
\hline Basic cuts & $6.81 \times 10^{-2}$ & $4.49 \times 10^{1}$ & $8.97 \times 10^{1}$ & $2.91 \times 10^{-1}$ & $5.05 \times 10^{-4}$ & $3.21 \times 10^{-1}$ \\
\hline Reconstruct scalars from $\tau \mathrm{s}$ & $3.14 \times 10^{-2}$ & $1.52 \times 10^{1}$ & $2.46 \times 10^{2}$ & $3.17 \times 10^{-2}$ & $1.20 \times 10^{-3}$ & $3.36 \times 10^{-1}$ \\
\hline Reconstruct scalars from $b \mathrm{~s}$ & $1.2 \times 10^{-2}$ & 2.47 & $1.06 \times 10^{-1}$ & 0.00 & $4.80 \times 10^{-3}$ & $4.10 \times 10^{-1}$ \\
\hline Cut on $M_{H^{0} A^{0}}$ & $6.99 \times 10^{-3}$ & $1.22 \times 10^{-1}$ & $2.06 \times 10^{-2}$ & 0.00 & $4.89 \times 10^{-2}$ & 1.00 \\
\hline Cut on $E_{T}$ & $5.04 \times 10^{-3}$ & $4.72 \times 10^{-2}$ & $5.88 \times 10^{-3}$ & 0.00 & $9.48 \times 10^{-2}$ & 1.18 \\
\hline Cascade enhanced & $5.11 \times 10^{-2}$ & - & - & - & $9.63 \times 10^{-1}$ & 10.7 \\
\hline$M_{\Delta}=190 \mathrm{GeV}$ & $H^{0} A^{0}\left(S_{0}\right)$ & $b \bar{b} \tau^{+} \tau^{-}$ & $\bar{c} \bar{c} \bar{b} W^{+} W^{-}$ & $Z h$ & $S / B$ & $\overline{\mathcal{S}(S, B)}$ \\
\hline Cross section at NLO & $2.47 \times 10^{-1}$ & $3.10 \times 10^{4}$ & $7.92 \times 10^{3}$ & $2.21 \times 10^{1}$ & $6.34 \times 10^{-6}$ & $6.86 \times 10^{-2}$ \\
\hline Basic cuts & $6.54 \times 10^{-3}$ & $4.49 \times 10^{1}$ & $8.97 \times 10^{1}$ & $2.91 \times 10^{-1}$ & $4.86 \times 10^{-5}$ & $3.09 \times 10^{-2}$ \\
\hline Reconstruct scalars from $\tau \mathrm{s}$ & $2.32 \times 10^{-3}$ & $4.60 \times 10^{-1}$ & $1.47 \times 10^{1}$ & $5.89 \times 10^{-3}$ & $1.53 \times 10^{-4}$ & $3.26 \times 10^{-2}$ \\
\hline Reconstruct scalars from $b \mathrm{~s}$ & $7.66 \times 10^{-4}$ & $2.06 \times 10^{-2}$ & 1.21 & 0.00 & $6.25 \times 10^{-4}$ & $3.78 \times 10^{-2}$ \\
\hline Cut on $M_{H^{0} A^{0}}$ & $6.34 \times 10^{-4}$ & $1.47 \times 10^{-3}$ & $9.03 \times 10^{-2}$ & 0.00 & $6.91 \times 10^{-3}$ & $1.14 \times 10^{-1}$ \\
\hline Cut on $E_{T}$ & $3.87 \times 10^{-4}$ & 0.00 & $2.64 \times 10^{-2}$ & 0.00 & $1.47 \times 10^{-2}$ & $1.30 \times 10^{-1}$ \\
\hline Cascade enhanced & $6.85 \times 10^{-3}$ & - & - & - & $2.60 \times 10^{-1}$ & 2.22 \\
\hline
\end{tabular}

TABLE II. Similar to TableI but for the $b \bar{b} \tau^{+} \tau^{-}$signal channel.

The different mass shift between $M_{\tau \tau}$ and $M_{b b}$ is owing to the missing neutrinos in $\tau$ decays resulting in a wider distribution of $M_{\tau \tau}$. For the reconstructed neutral scalars, we further adopt similar cuts on $M_{H^{0} A^{0}}$ and $E_{T}$ as in the $b \bar{b} \gamma \gamma$ channel:

$$
M_{H^{0} A^{0}}>2 M_{\Delta}+70 \mathrm{GeV}, E_{T}>2 M_{\Delta}-80 \mathrm{GeV}
$$

Both $M_{H^{0} A^{0}}$ and $E_{T}$ cuts are reduced by $20 \mathrm{GeV}$ compared with the $b \bar{b} \gamma \gamma$ channel, which again results from neutrinos in the final state. The corresponding results are summarized in Table $\mathrm{II}$

The $b \bar{b} \tau^{+} \tau^{-}$is also promising for $M_{\Delta}=130 \mathrm{GeV}$ even without enhancement from cascade decays. The final significance is 12.4 and the corresponding number of signal events is 309 for LHC14@3000. 
Including the cascade enhancement, the significance is improved to 51.6, which is even better than the $b \bar{b} \gamma \gamma$ signal. For $M_{\Delta}=160 \mathrm{GeV}$, the biggest challenge is also the small production cross section of the signal. But in the most optimistic case, the cascade decays can increase the signal by a factor of 10.1, making this channel feasible. Finally, neutral scalars as heavy as $190 \mathrm{GeV}$ are difficult to detect at LHC14 in this channel.

\section{C. $\quad b \bar{b} W^{+} W^{-}$signal channel}

It is difficult to search for the SM Higgs pair production in this channel due to missing energy brought about by neutrinos in leptonic decays of the $W$ boson, which makes one of the two Higgs bosons not fully reconstructible [36, 57]. The situation is ameliorated in our scenario because, the production rate of $H^{0} A^{0}$ can be an order of magnitude larger than that of $h h$ and the di- $W$ decay branching ratio of $H^{0}$ can also be larger than $h$ in the vast parameter space. This considerably improves the signal events and partially compensates the deficiency of the detection capability.

With both $W$ 's decaying leptonically, the final state appears as $b \bar{b} \ell^{+} \ell^{-} E_{T}$. The dominant SM backgrounds are as follows:

$$
t \bar{t}: p p \rightarrow t \bar{t} \rightarrow b W^{+} \bar{b} W^{-} \rightarrow b \bar{b} \ell^{+} \ell^{-} E_{T}
$$

As before, the QCD correction is included by a multiplicative $K$-factor of 1.35 for the $t \bar{t}$ production [77]. We pick up the events that include exactly one $b$-jet pair and one opposite-sign lepton pair and filter them with the basic cuts:

$$
\begin{array}{r}
p_{T}^{b}>30 \mathrm{GeV}, p_{T}^{\ell}>20 \mathrm{GeV},\left|\eta_{b, \ell}\right|<2.4, \\
\Delta R_{b b, b \ell, \ell \ell}>0.4, E_{T}>20 \mathrm{GeV} .
\end{array}
$$

The separation and invariant mass of the $b$-jet pair are required to fulfill

$$
\Delta R_{b b}<2.5,\left|M_{b b}-M_{\Delta}\right|<15 \mathrm{GeV}
$$

For the lepton pair, we reconstruct the transverse cluster mass $M_{C}^{\ell \ell}$ :

$$
M_{C}^{\ell \ell}=\sqrt{\left(\sqrt{p_{T, \ell \ell}^{2}+M_{\ell \ell}^{2}}+E_{T}\right)^{2}+\left(\vec{p}_{T, \ell \ell}+\vec{E}_{T}\right)^{2}} .
$$

The distributions of $M_{C}^{\ell \ell}, \Delta R_{\ell \ell}$, and $E_{T}$ are shown in Fig. 14. The peak of $M_{C}^{\ell \ell}$ is always lower than $M_{\Delta}$ by about $30-40 \mathrm{GeV}$, and the lepton separation $\Delta R_{\ell \ell}$ in the signal is much smaller than in the $t \bar{t}$ background. Accordingly, we set a wide window on $M_{C}^{\ell \ell}$ while tightening up the cuts on $\Delta R_{\ell \ell}$ and $E_{T}$ :

$$
M_{\Delta}-80 \mathrm{GeV}<M_{C}^{\ell \ell}<M_{\Delta}, \Delta R_{\ell \ell}<1.2, E_{T}>0.9 M_{\Delta}
$$



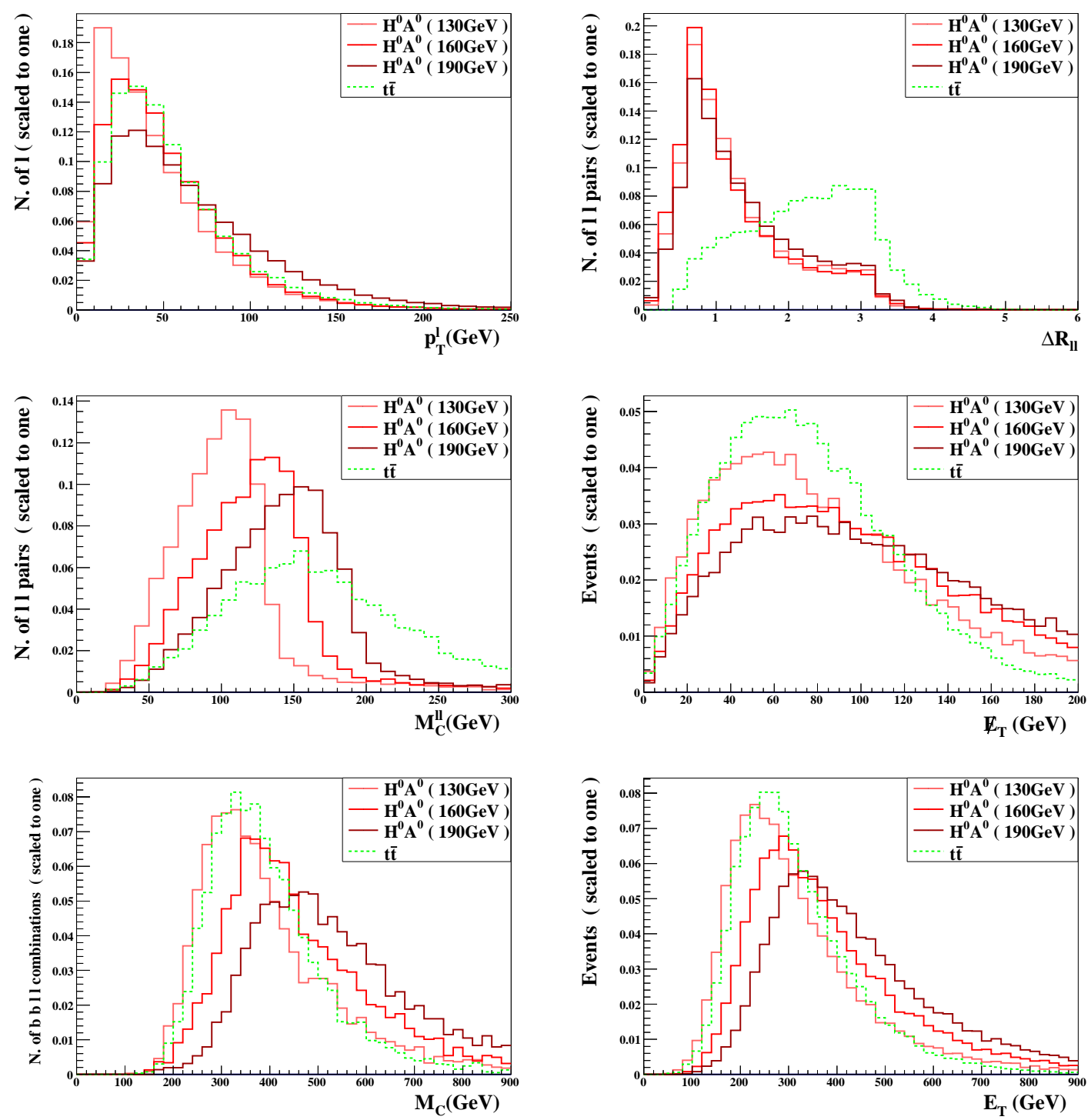

FIG. 14. Distributions of $p_{T}^{\ell}, \Delta R_{\ell \ell}, M_{C}^{\ell \ell}, E_{T}, M_{C}$, and $E_{T}$ for the signal $b \bar{b} \ell^{+} \ell^{-} E_{T}$ and its backgrounds before applying any cuts at $\mathrm{LHC} 14$.

We find that $M_{C}^{\ell \ell}$ is least efficient around $M_{\Delta} \sim 190 \mathrm{GeV}$, where the peak of $M_{C}^{\ell \ell}$ for the $t \bar{t}$ background is around $150 \mathrm{GeV}$. The very tight cuts on $\Delta R_{\ell \ell}$ and $E_{T}$ are sufficient to suppress the background by 1 or 2 orders of magnitude, while keeping the number of signal events as large as possible. We further combine the $b$-jet pair and the lepton pair into a cluster and construct the transverse cluster mass:

$$
M_{C}=\sqrt{\left(\sqrt{p_{T, b b \ell \ell}^{2}+M_{b b \ell \ell}^{2}}+E_{T}\right)^{2}-\left(\vec{p}_{T, b b \ell \ell}+\vec{E}_{T}\right)^{2}}
$$

which is an analog of $M_{H^{0} A^{0}}$ in the previous subsection. The distribution of $M_{C}$ is displayed in Fig. 14, which is very similar to that of $M_{H^{0} A^{0}}$ in the $b \bar{b} \gamma \gamma$ channel. Although it looks from the $M_{C}$ distributions 


\begin{tabular}{|c|c|c|c|c|}
\hline$M_{\Delta}=130 \mathrm{GeV}$ & $H^{0} A^{0}\left(S_{0}\right)$ & $t \bar{t}$ & $S / B$ & $\mathcal{S}(S, B)$ \\
\hline Cross section at NLO & 3.91 & $2.38 \times 10^{4}$ & $1.69 \times 10^{-4}$ & 1.41 \\
\hline Basic cuts & 1.51 & $4.04 \times 10^{3}$ & $3.74 \times 10^{-4}$ & 1.30 \\
\hline Reconstruct scalars from $b s$ & $3.29 \times 10^{-1}$ & $3.35 \times 10^{2}$ & $9.82 \times 10^{-4}$ & 0.984 \\
\hline Cut on $M_{C}^{\ell \ell}$ & $3.21 \times 10^{-1}$ & $2.14 \times 10^{2}$ & $1.50 \times 10^{-3}$ & 1.20 \\
\hline Cut on $\Delta R_{\ell \ell}$ & $2.64 \times 10^{-1}$ & $9.26 \times 10^{1}$ & $2.85 \times 10^{-3}$ & 1.50 \\
\hline Cut on $E_{T}$ & $8.45 \times 10^{-2}$ & $1.48 \times 10^{1}$ & $5.71 \times 10^{-3}$ & 1.20 \\
\hline Cut on $M_{C}$ & $3.30 \times 10^{-2}$ & $1.69 \times 10^{-1}$ & $1.95 \times 10^{-1}$ & 4.26 \\
\hline Cut on $E_{T}$ & $3.19 \times 10^{-2}$ & $1.47 \times 10^{-1}$ & $2.17 \times 10^{-1}$ & 4.41 \\
\hline Casc & $1.40 \times 10^{-1}$ & - & $9.53 \times 10^{-1}$ & 17.7 \\
\hline$M_{\Delta}=160 \mathrm{GeV}$ & $H^{0} A^{0}\left(S_{0}\right)$ & $t \bar{t}$ & $S / B$ & $\mathcal{S}(S, 1$ \\
\hline Cross se & 4.95 & $2.38 \times 10^{4}$ & $2.13 \times 10^{-4}$ & 1.78 \\
\hline Basic cuts & 2.13 & $4.04 \times 10^{3}$ & $5.27 \times 10^{-4}$ & 1.84 \\
\hline Reconstruct scalars from $b \mathrm{~s}$ & $4.25 \times 10^{-1}$ & $2.68 \times 10^{2}$ & $1.59 \times 10^{-3}$ & 1.42 \\
\hline Cut on $M_{C}^{\ell \ell}$ & $3.97 \times 10^{-1}$ & $1.89 \times 10^{2}$ & $2.10 \times 10^{-3}$ & 1.58 \\
\hline Cut on $\Delta R_{\ell \ell}$ & $3.21 \times 10^{-1}$ & $7.04 \times 10^{1}$ & $4.56 \times 10^{-3}$ & 2.09 \\
\hline Cut on $E_{T}$ & $9.47 \times 10^{-2}$ & 4.29 & $2.21 \times 10^{-2}$ & 2.50 \\
\hline Cut on $M_{C}$ & $3.28 \times 10^{-2}$ & $4.74 \times 10^{-2}$ & $6.92 \times 10^{-1}$ & 7.50 \\
\hline Cut on $E_{T}$ & $3.02 \times 10^{-2}$ & $3.62 \times 10^{-2}$ & $8.34 \times 10^{-1}$ & 7.78 \\
\hline $\mathrm{Cas}$ & $1.01 \times 10^{-1}$ & - & 3.24 & 23.2 \\
\hline$M_{\Delta}=190 \mathrm{GeV}$ & $H^{0} A^{0}\left(S_{0}\right)$ & $t \bar{t}$ & $S / B$ & $\mathcal{O}(S$, \\
\hline Cross section at NLO & 1.19 & $2.38 \times 10^{4}$ & $5.00 \times 10^{-5}$ & 0.424 \\
\hline Basic cuts & $6.44 \times 10^{-1}$ & $4.04 \times 10^{3}$ & $1.59 \times 10^{-4}$ & 0.554 \\
\hline Reconstruct scalars from $b \mathrm{~s}$ & $1.36 \times 10^{-1}$ & $2.26 \times 10^{2}$ & $6.02 \times 10^{-4}$ & 0.495 \\
\hline Cut on $M_{C}^{\ell \ell}$ & $1.27 \times 10^{-1}$ & $1.79 \times 10^{2}$ & $7.09 \times 10^{-4}$ & 0.520 \\
\hline Cut on $\Delta R_{\ell \ell}$ & $9.70 \times 10^{-2}$ & $6.05 \times 10^{1}$ & $1.60 \times 10^{-3}$ & 0.683 \\
\hline Cut on $\mathscr{E}_{T}$ & $2.57 \times 10^{-2}$ & 1.62 & $1.59 \times 10^{-2}$ & 1.10 \\
\hline Cut on $M_{C}$ & $8.85 \times 10^{-3}$ & $1.89 \times 10^{-2}$ & $4.68 \times 10^{-1}$ & 3.29 \\
\hline Cut on $E_{T}$ & $8.37 \times 10^{-3}$ & $1.40 \times 10^{-2}$ & $5.98 \times 10^{-1}$ & 3.56 \\
\hline Cascade enhanced & $2.69 \times 10^{-2}$ & - & 1.92 & 10.1 \\
\hline
\end{tabular}

TABLE III. Similar to Table I but for the $b \bar{b} \ell^{+} \ell^{-} E_{T}$ signal channel.

(before any cuts are made) that the $t \bar{t}$ background has a large overlap with the signal, the cuts on $M_{C}^{\ell \ell}$, $\Delta R_{\ell \ell}$, and $E_{T}$ actually modify them remarkably, so that a further cut on $M_{C}$ could improve the significance 

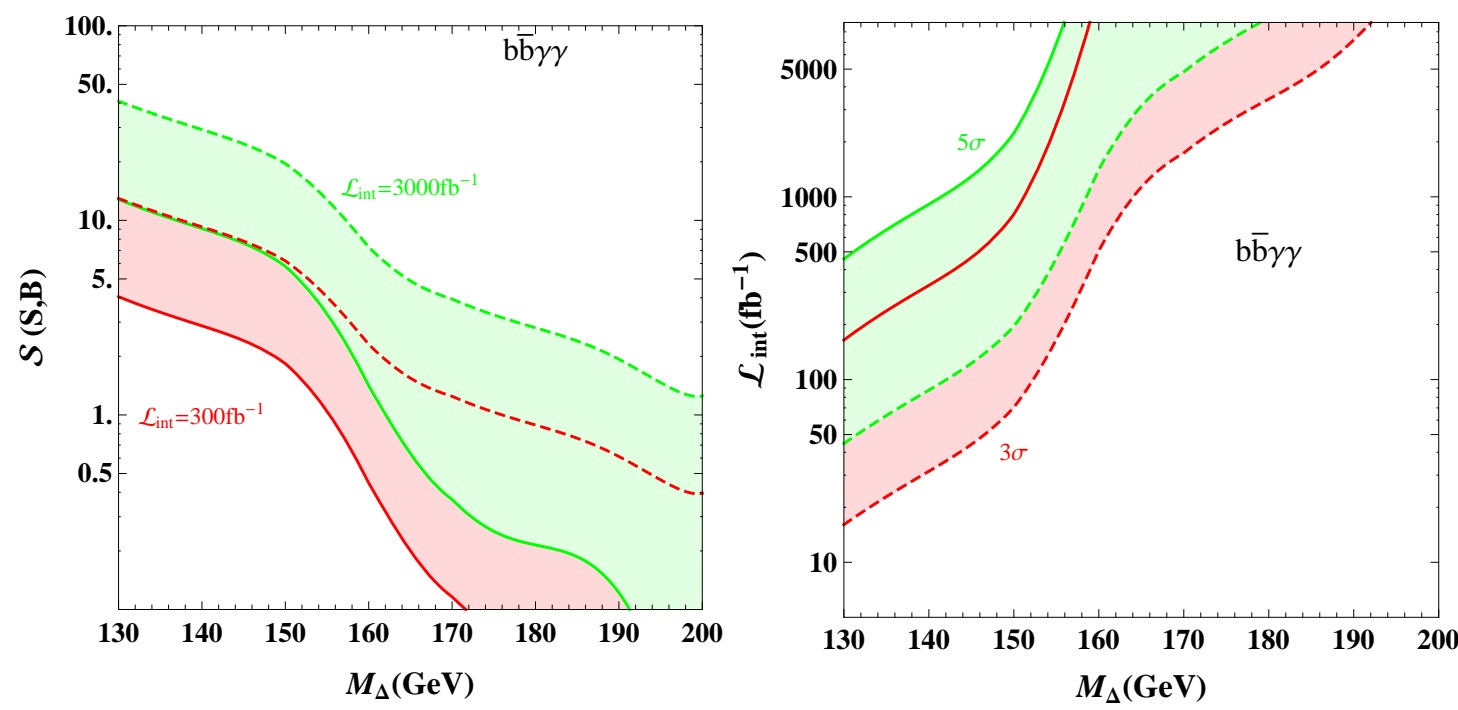

FIG. 15. Left: Significance $\mathcal{S}(S, B)$ of the $b \bar{b} \gamma \gamma$ channel versus $M_{\Delta}$ reachable at LHC14@300 (red region) and LHC14@3000 (green). Right: Required luminosity to reach a $3 \sigma$ (red region) and $5 \sigma$ (green) significance in the $b \bar{b} \gamma \gamma$ channel versus $M_{\Delta}$ at LHC14. The solid line corresponds to the signal from $X_{0}$ alone, and the dashed line corresponds to the total signal including cascade enhancement.

efficiently. We apply a cut on $M_{C}$ as we did with $M_{H^{0} A^{0}}$, as well as one on $E_{T}$ :

$$
M_{C}>2 M_{\Delta}+90 \mathrm{GeV}, E_{T}>2 M_{\Delta}-60 \mathrm{GeV} .
$$

The results following the cutflow are summarized in Table III. For $M_{\Delta}=130 \mathrm{GeV}$, the final significance is 4.41 (17.7) without (with) cascade enhancement. With cascade enhancement this should be enough to discover the neutral scalars. The signal channel is more promising for $M_{\Delta}=160 \mathrm{GeV}$ due to a slightly larger cross section and higher cut efficiencies. The final significance is 7.78 (23.2), which is also better than the $b \bar{b} \gamma \gamma$ and $b \bar{b} \tau^{+} \tau^{-}$channels with the same mass. Finally, for $M_{\Delta}=190 \mathrm{GeV}$, the significance becomes 3.56 (10.1). Therefore, for our benchmark model, the only promising signal for such heavy neutral scalars $(\sim 190 \mathrm{GeV})$ comes from the $b \bar{b} W^{+} W^{-}$channel.

\section{Observability}

Based on our elaborate analysis of signal channels in Secs. IV A $\sqrt{\mathrm{IVC}}$, we examine the observability of the neutral scalars $H_{0}, A_{0}$ in the mass region $130 \sim 200 \mathrm{GeV}$ by adopting essentially the same cuts as before. In the left panel of Figs. 15, 16, and 17 we present the significance $\mathcal{S}(S, B)$ as a function of $M_{\Delta}$ in the three signal channels $b \bar{b} \gamma \gamma, b \bar{b} \tau^{+} \tau^{-}$, and $b \bar{b} \ell^{+} \ell^{-} E_{T}$ that is reachable for LHC14@300 and LHC14@3000, respectively. The required luminosity to achieve a $3 \sigma$ and $5 \sigma$ significance is displayed in 

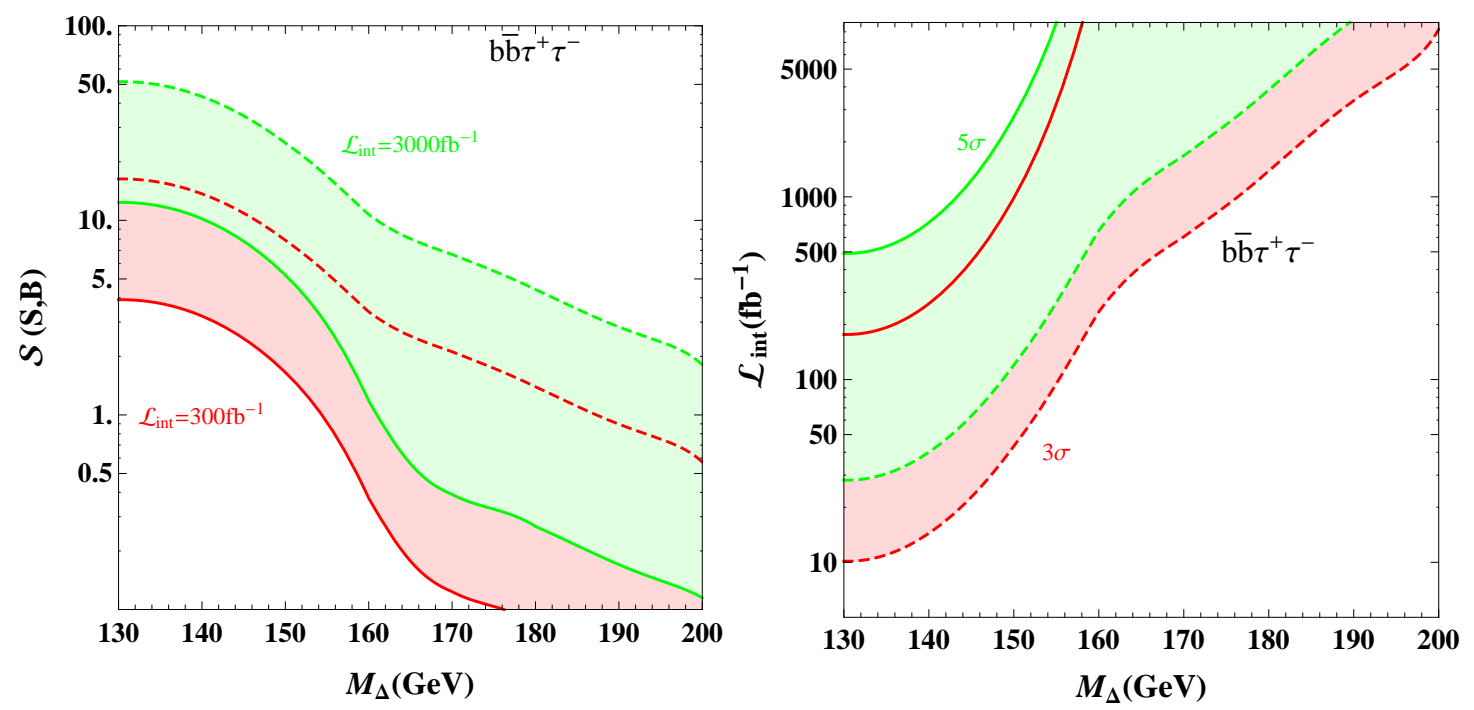

FIG. 16. Same as Fig. 15, but for the $b \bar{b} \tau^{+} \tau^{-}$channel.

the right panel of the figures. As was done in our previous analysis, the effect of cascade enhancement is included by a factor $S / S_{0}$ in the final results.

As shown in Figs. 15 and 16 , both the $b \bar{b} \gamma \gamma$ and $b \bar{b} \tau^{+} \tau^{-}$channel are typically sensitive to the low-mass region $\left(M_{\Delta} \lesssim 160 \mathrm{GeV}\right)$. In the absence of cascade enhancement, the $3 \sigma$ significance would never be reached for $M_{\Delta} \gtrsim 138(142) \mathrm{GeV}$ in the $b \bar{b} \gamma \gamma\left(b \bar{b} \tau^{+} \tau^{-}\right)$channel for LHC14@300. However, a cascade enhancement of $S / S_{0} \sim 4-6$ (as can be seen from Fig. 11) in this mass region can greatly improve the observability, pushing the $3 \sigma$ mass limit up to $157(162) \mathrm{GeV}$ in the $b \bar{b} \gamma \gamma\left(b \bar{b} \tau^{+} \tau^{-}\right)$channel. Moreover, with cascade enhancement, one has a good chance to reach a $5 \sigma$ significance if $M_{\Delta} \lesssim 153(155) \mathrm{GeV}$. In other words, the cascade enhancement significantly reduces the required luminosity. For instance, to achieve a $3 \sigma$ and $5 \sigma$ significance in the $b \bar{b} \gamma \gamma\left(b \bar{b} \tau^{+} \tau^{-}\right)$channel with $M_{\Delta}=130 \mathrm{GeV}$, the required luminosity is as low as $16(10) \mathrm{fb}^{-1}$ and $42(27) \mathrm{fb}^{-1}$ at LHC14, respectively. The $b \bar{b} \tau^{+} \tau^{-}$channel is more promising, thanks to a relatively larger production rate.

At the future LHC14 with $3000 \mathrm{fb}^{-1}$ data, the heavier mass region can also be probed. With a maximal cascade enhancement, the $3 \sigma$ and $5 \sigma$ mass reach is pushed to 177 and $164 \mathrm{GeV}$, respectively, in the $b \bar{b} \gamma \gamma$ channel, which should be compared to 156 and $151 \mathrm{GeV}$ in the absence of enhancement. For the $b \bar{b} \tau^{+} \tau^{-}$ channel, the enhancement factor $S / S_{0}$ can reach about 18 above the $W$-pair threshold, upshifting the $3 \sigma$ and $5 \sigma$ mass reach to 189 and $177 \mathrm{GeV}$, respectively, from 154 and $150 \mathrm{GeV}$ without the enhancement.

The $b \bar{b} \ell^{+} \ell^{-} E_{T}$ channel shown in Fig. 17 is more special, compared with $b \bar{b} \gamma \gamma$ and $b \bar{b} \tau^{+} \tau^{-}$. It is relatively more sensitive to a higher mass between $150-180 \mathrm{GeV}$, where the decay mode $H^{0} \rightarrow W^{+} W^{-}$ dominates, while its observability deteriorates for $M_{\Delta}<150 \mathrm{GeV}$ due to phase-space suppression in the 

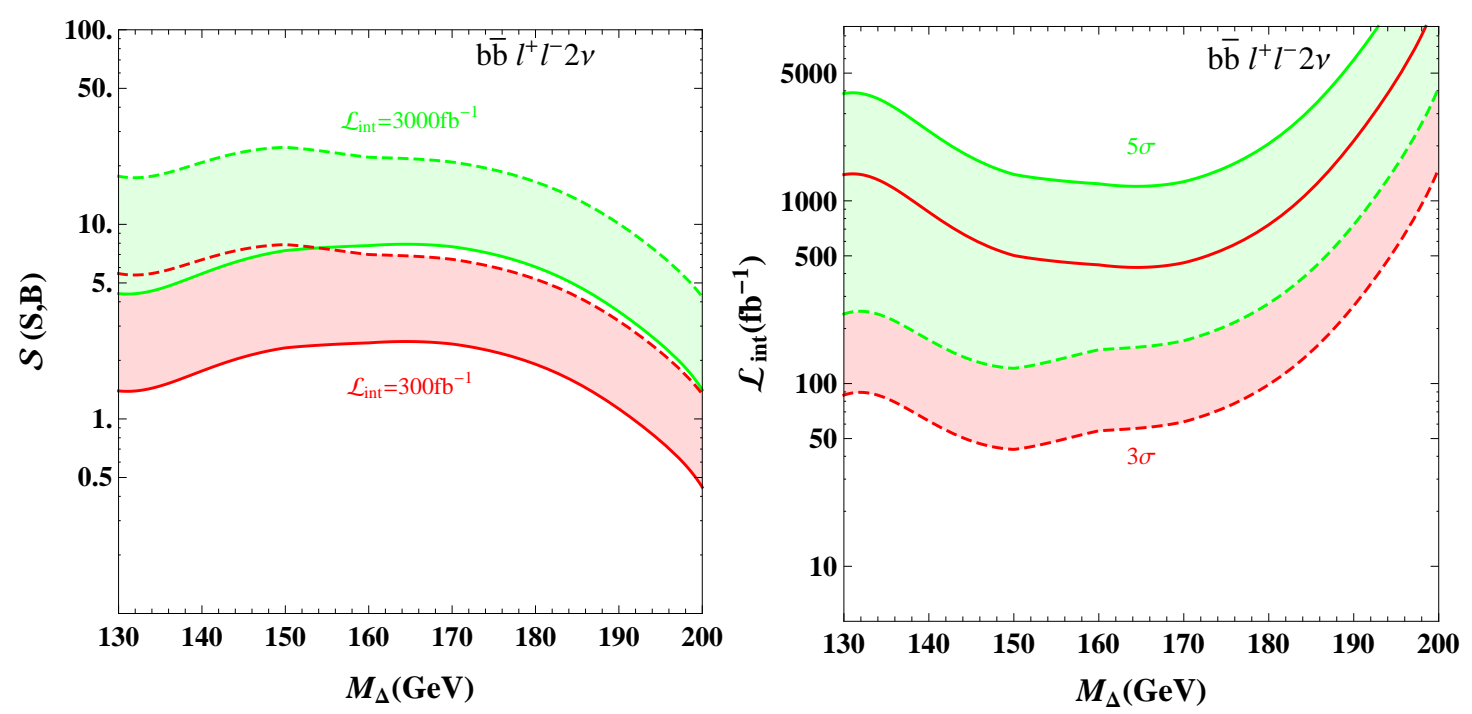

FIG. 17. Same as Fig. 15, but for the $b \bar{b} \ell^{+} \ell^{-} E_{T}$ channel.

decay. The cascade enhancement $S / S_{0}$ at our benchmark point 9 is typically $3-4$ in the mass region 130 $200 \mathrm{GeV}$, and decreases as $M_{\Delta}$ increases. For LHC14@300, the $3 \sigma$ and $5 \sigma$ mass reach is, respectively, 190 and $181 \mathrm{GeV}$ with maximal cascade enhancement. These limits would just increase by $2-3 \mathrm{GeV}$ for LHC14@3000 if there were no cascade enhancement, while with cascade enhancement the $5 \sigma$ limit, for instance, is pushed up to $200 \mathrm{GeV}$. Finally, a $3 \sigma$ or $5 \sigma$ reach in the mass region $150-180 \mathrm{GeV}$ requires an integrated luminosity of $50 \mathrm{fb}^{-1}\left(450 \mathrm{fb}^{-1}\right)$ or $150 \mathrm{fb}^{-1}\left(1300 \mathrm{fb}^{-1}\right)$ with (without) cascade enhancement.

\section{DISCUSSIONS AND CONCLUSIONS}

In this paper, we have systematically investigated the LHC phenomenology of neutral scalar pair production in the negative scenario of the type II seesaw model. To achieve this goal, we first examined the decay properties of the neutral scalars $H_{0} / A_{0}$ and found that the scalar self-couplings $\lambda_{i}$ have a great impact on the branching ratios of $H^{0} / A^{0}$. The coupling $\lambda_{4}$ is important for tree-level decays of $H^{0}$ and $A^{0}$, while one-loop-induced decays of $H^{0}$ further depend on $\lambda_{2}$ and $\lambda_{3}$. We found that the decay $H^{0} \rightarrow W^{+} W^{-}$ could dominate for $2 M_{W}<M_{H^{0}}<2 M_{h}$ with $\lambda_{4}<0$, while it can be neglected once $M_{H^{0}}$ is above the light scalar pair threshold $2 M_{h}$. Moreover, the branching ratios of the decays $H^{0} \rightarrow \gamma \gamma, Z \gamma$ can cross 3 orders of magnitude when varying the couplings $\lambda_{i}$, and there exist zero points for the $H^{0} Z Z, H^{0} h h$, and $A^{0} Z h$ couplings.

The cross section of the Drell-Yan process $p p \rightarrow Z^{*} \rightarrow H^{0} A^{0}$ for $M_{\Delta}<200 \mathrm{GeV}$ is much larger than that of the SM Higgs pair production driven by gluon fusion. In this paper, we studied the contributions 
to $H^{0} / A^{0}$ production from cascade decays of the charged scalars $H^{ \pm}$and $H^{ \pm \pm}$. There are actually three different states for the neutral scalar pair: $H^{0} A^{0}, H^{0} H^{0}$, and $A^{0} A^{0}$. Here, $H^{0} H^{0}$ and $A^{0} A^{0}$ can only arise from cascade decays of charged scalars, and their production rates always stay the same to a good approximation. Further, for a fixed value of $M_{\Delta}$, cascade enhancement is determined by the variables $v_{\Delta}$ and $\Delta M$. By tuning these two variables, the associated production rate of $H^{0} A^{0}$ can be maximally enhanced by about a factor of 3 , while those of the $H^{0} H^{0}$ and $A^{0} A^{0}$ pair production can reach the value of $H^{0} A^{0}$ production through the pure Drell-Yan process.

We implemented detailed collider simulations of the associated $H^{0} A^{0}$ production for three typical signal channels $\left(b \bar{b} \gamma \gamma, b \bar{b} \tau^{+} \tau^{-}\right.$, and $b \bar{b} W^{+} W^{-}$with both $W$ 's decaying leptonically). The enhancement from cascade decays of charged scalars is quantified by a multiplicative factor $S / S_{0}$. Due mainly to a larger production rate, all three channels are more promising than the SM Higgs pair case. If there were no cascade enhancement, the $5 \sigma$ mass reach of the $b \bar{b} \gamma \gamma, b \bar{b} \tau^{+} \tau^{-}$, and $b \bar{b} \ell^{+} \ell^{-} E_{T}$ channels would be, respectively, 151, 150, and $180 \mathrm{GeV}$ for LHC14@3000. The cascade enhancement pushes these limits up to 164, 177, and $200 \mathrm{GeV}$. The $b \bar{b} \gamma \gamma$ and $b \bar{b} \tau^{+} \tau^{-}$channels are more promising in the mass region below about $150 \mathrm{GeV}$, and the required luminosities for $5 \sigma$ significance are $42 \mathrm{fb}^{-1}$ and $27 \mathrm{fb}^{-1}$, respectively, at our benchmark point. Compared with these two channels, the $b \bar{b} \ell^{+} \ell^{-} E_{T}$ channel is more advantageous in the relatively higher mass region $150-200 \mathrm{GeV}$, and the required luminosity for $5 \sigma$ significance is about $150 \mathrm{fb}^{-1}$ with maximal cascade enhancement. Needless to say, for the purpose of a full investigation on the impact of heavy neutral scalars on the SM Higgs pair production, more sophisticated simulations are necessary. We hope that this work may shed some light on further studies in both the phenomenological and experimental communities.

\section{ACKNOWLEDGMENTS}

This work was supported in part by the Grants No. NSFC-11025525, No. NSFC-11575089 and by the CAS Center for Excellence in Particle Physics (CCEPP).

[1] Z. L. Han, R. Ding, and Y. Liao, Phys. Rev. D 91, 093006 (2015) [arXiv:1502.05242 [hep-ph]].

[2] G. Aad et al. [ATLAS Collaboration], Phys. Lett. B 726, 88 (2013); Phys. Lett. B 734, 406 (2014) [arXiv:1307.1427[hep-ex]].

[3] S. Chatrchyan et al. [CMS Collaboration], JHEP 1306, 081 (2013) arXiv:1303.4571 [hep-ex]].

[4] G. Aad et al. [ATLAS Collaboration], Phys. Lett. B 716, 1 (2012) arXiv:1207.7214 [hep-ex]]. 
[5] S. Chatrchyan et al. [CMS Collaboration], Phys. Lett. B 716, 30 (2012) [arXiv:1207.7235 [hep-ex]].

[6] T. Plehn, M. Spira, and P. M. Zerwas, Nucl. Phys. B 479, 46 (1996) [Erratum-ibid. B 531, 655 (1998)] [hepph/9603205].

[7] S. Dawson, S. Dittmaier, and M. Spira, Phys. Rev. D 58, 115012 (1998) [hep-ph/9805244].

[8] A. Djouadi, W. Kilian, M. Muhlleitner, and P. M. Zerwas, Eur. Phys. J. C 10, 45 (1999) [hep-ph/9904287].

[9] U. Baur, T. Plehn, and D. L. Rainwater, Phys. Rev. D 67, 033003 (2003) [hep-ph/0211224].

[10] E. Asakawa, D. Harada, S. Kanemura, Y. Okada, and K. Tsumura, Phys. Rev. D 82, 115002 (2010) [arXiv:1009.4670 [hep-ph]].

[11] M. J. Dolan, C. Englert, and M. Spannowsky, JHEP 1210, 112 (2012) arXiv:1206.5001 [hep-ph]].

[12] A. Papaefstathiou, L. L. Yang, and J. Zurita, Phys. Rev. D 87, no. 1, 011301 (2013) [arXiv:1209.1489 [hep-ph]].

[13] F. Goertz, A. Papaefstathiou, L. L. Yang, and J. Zurita, JHEP 1306, 016 (2013) [arXiv:1301.3492 [hep-ph]].

[14] R. S. Gupta, H. Rzehak, and J. D. Wells, Phys. Rev. D 88, 055024 (2013) [arXiv:1305.6397 [hep-ph]].

[15] A. J. Barr, M. J. Dolan, C. Englert, and M. Spannowsky, Phys. Lett. B 728, 308 (2014) arXiv:1309.6318 [hep$\mathrm{ph}]]$.

[16] D. de Florian and J. Mazzitelli, Phys. Rev. Lett. 111, 201801 (2013) [arXiv:1309.6594 [hep-ph]].

[17] M. J. Dolan, C. Englert, N. Greiner, and M. Spannowsky, Phys. Rev. Lett. 112, 101802 (2014) arXiv:1310.1084 [hep-ph]].

[18] V. Barger, L. L. Everett, C. B. Jackson, and G. Shaughnessy, Phys. Lett. B 728, 433 (2014) arXiv:1311.2931 [hep-ph]].

[19] C. Englert, F. Krauss, M. Spannowsky, and J. Thompson, Phys. Lett. B 743, 93 (2015) arXiv:1409.8074 [hep$\mathrm{ph}]]$.

[20] T. Liu and H. Zhang, arXiv:1410.1855 [hep-ph].

[21] D. E. Ferreira de Lima, A. Papaefstathiou, and M. Spannowsky, JHEP 1408, 030 (2014) arXiv:1404.7139 [hep-ph]].

[22] A. J. Barr, M. J. Dolan, C. Englert, D. E. F. de Lima, and M. Spannowsky, arXiv:1412.7154 [hep-ph].

[23] M. J. Dolan, C. Englert, and M. Spannowsky, Phys. Rev. D 87, no. 5, 055002 (2013) [arXiv:1210.8166 [hep-ph]].

[24] A. Arhrib, R. Benbrik, C. -H. Chen, R. Guedes, and R. Santos, JHEP 0908, 035 (2009) arXiv:0906.0387 [hep$\mathrm{ph}]]$.

[25] N. Craig, J. Galloway, and S. Thomas, arXiv:1305.2424 [hep-ph].

[26] B. Hespel, D. Lopez-Val, and E. Vryonidou, JHEP 1409, 124 (2014) [arXiv:1407.0281 [hep-ph]].

[27] G. D. Kribs and A. Martin, Phys. Rev. D 86, 095023 (2012) [arXiv:1207.4496 [hep-ph]].

[28] J. Cao, Z. Heng, L. Shang, P. Wan, and J. M. Yang, JHEP 1304, 134 (2013) arXiv:1301.6437 [hep-ph]].

[29] D. T. Nhung, M. Muhlleitner, J. Streicher, and K. Walz, JHEP 1311, 181 (2013) [arXiv:1306.3926 [hep-ph]].

[30] U. Ellwanger, JHEP 1308, 077 (2013) [arXiv:1306.5541 [hep-ph]].

[31] B. Bhattacherjee and A. Choudhury, Phys. Rev. D 91, 073015 (2015) [arXiv:1407.6866 [hep-ph]].

[32] N. D. Christensen, T. Han, and T. Li, Phys. Rev. D 86, 074003 (2012) arXiv:1206.5816 [hep-ph]].

[33] L. Wu, J. M. Yang, C. P. Yuan, and M. Zhang, Phys. Lett. B 747, 378 (2015) [arXiv:1504.06932 [hep-ph]]. 
[34] J. Cao, D. Li, L. Shang, P. Wu, and Y. Zhang, JHEP 1412, 026 (2014) [arXiv:1409.8431 [hep-ph]].

[35] C. Han, X. Ji, L. Wu, P. Wu, and J. M. Yang, JHEP 1404, 003 (2014) [arXiv:1307.3790 [hep-ph]].

[36] M. Gouzevitch, A. Oliveira, J. Rojo, R. Rosenfeld, G. P. Salam, and V. Sanz, JHEP 1307, 148 (2013) [arXiv:1303.6636 [hep-ph]].

[37] J. M. No and M. Ramsey-Musolf, Phys. Rev. D 89, 095031 (2014) [arXiv:1310.6035 [hep-ph]].

[38] R. Grober and M. Muhlleitner, JHEP 1106, 020 (2011) [arXiv:1012.1562 [hep-ph]].

[39] M. Gillioz, R. Grober, C. Grojean, M. Muhlleitner, and E. Salvioni, JHEP 1210, 004 (2012) [arXiv:1206.7120 [hep-ph]].

[40] J. Liu, X. P. Wang, and S. h. Zhu, arXiv:1310.3634 [hep-ph].

[41] A. Arhrib, R. Benbrik, R. B. Guedes, and R. Santos, Phys. Rev. D 78, 075002 (2008) arXiv:0805.1603 [hep$\mathrm{ph}]]$.

[42] Z. Heng, L. Shang, Y. Zhang, and J. Zhu, JHEP 1402, 083 (2014) [arXiv:1312.4260 [hep-ph]].

[43] S. Dawson, E. Furlan, and I. Lewis, Phys. Rev. D 87, 014007 (2013) [arXiv:1210.6663 [hep-ph]].

[44] C. Y. Chen, S. Dawson, and I. M. Lewis, Phys. Rev. D 90, 035016 (2014) [arXiv:1406.3349 [hep-ph]].

[45] C. O. Dib, R. Rosenfeld, and A. Zerwekh, JHEP 0605, 074 (2006) [hep-ph/0509179].

[46] B. Yang, Z. Liu, N. Liu, and J. Han, Eur. Phys. J. C 74, 3203 (2014) arXiv:1408.4295 [hep-ph]].

[47] C. Y. Chen, S. Dawson, and I. M. Lewis, Phys. Rev. D 91, 035015 (2015) [arXiv:1410.5488 [hep-ph]].

[48] R. Contino, M. Ghezzi, M. Moretti, G. Panico, F. Piccinini, and A. Wulzer, JHEP 1208, 154 (2012) [arXiv:1205.5444[hep-ph]].

[49] K. Nishiwaki, S. Niyogi, and A. Shivaji, JHEP 1404, 011 (2014) [arXiv:1309.6907 [hep-ph]].

[50] N. Liu, S. Hu, B. Yang, and J. Han, JHEP 1501, 008 (2015) [arXiv:1408.4191 [hep-ph]].

[51] S. Dawson, A. Ismail, and I. Low, Phys. Rev. D 91, 115008 (2015) [arXiv:1504.05596 [hep-ph]].

[52] A. Azatov, R. Contino, G. Panico, and M. Son, Phys. Rev. D 92, 035001 (2015) [arXiv:1502.00539 [hep-ph]].

[53] F. Goertz, A. Papaefstathiou, L. L. Yang, and J. Zurita, JHEP 1504, 167 (2015) [arXiv:1410.3471][hep-ph]].

[54] A. Pierce, J. Thaler, and L. -T. Wang, JHEP 0705, 070 (2007) [hep-ph/0609049].

[55] Z. Kang, P. Ko, and J. Li, arXiv:1504.04128 [hep-ph].

[56] H. J. He, J. Ren, and W. Yao, arXiv:1506.03302 [hep-ph].

[57] J. Baglio, A. Djouadi, R. Gröber, M. M. Mühlleitner, J. Quevillon, and M. Spira, JHEP 1304, 151 (2013) [arXiv:1212.5581[hep-ph]].

[58] S. Dawson et al., arXiv:1310.8361 [hep-ex].

[59] T. P. Cheng and L. F. Li, Phys. Rev. D 22, 2860 (1980); J. Schechter and J. W. F. Valle, Phys. Rev. D 22, 2227 (1980); G. Lazarides, Q. Shafi, and C. Wetterich, Nucl. Phys. B 181, 287 (1981); R. N. Mohapatra and G. Senjanovic, Phys. Rev. D 23, 165 (1981); M. Magg and C. Wetterich, Phys. Lett. B 94, 61 (1980).

[60] A. Arhrib et al., Phys. Rev. D 84, 095005 (2011) arXiv:1105.1925 [hep-ph]].

[61] M. Aoki, S. Kanemura, M. Kikuchi, and K. Yagyu, Phys. Rev. D 87, 015012 (2013) [arXiv:1211.6029 [hep-ph]]; S. Kanemura and K. Yagyu, Phys. Rev. D 85, 115009 (2012) [arXiv:1201.6287 [hep-ph]].

[62] M. Aoki, S. Kanemura, and K. Yagyu, Phys. Rev. D 85, 055007 (2012) arXiv:1110.4625 [hep-ph]]. 
[63] M. Chabab, M. C. Peyranere, and L. Rahili, Phys. Rev. D 90, 035026 (2014) arXiv:1407.1797 [hep-ph]].

[64] A. Djouadi, Phys. Rep. 459, 1 (2008) [hep-ph/0503173].

[65] P. Fileviez Perez, T. Han, G. -y. Huang, T. Li, and K. Wang, Phys. Rev. D 78, 015018 (2008) [arXiv:0805.3536 [hep-ph]].

[66] A. Melfo, M. Nemevsek, F. Nesti, G. Senjanovic, and Y. Zhang, Phys. Rev. D 85, 055018 (2012) arXiv:1108.4416 [hep-ph]].

[67] E. J. Chun and P. Sharma, Phys. Lett. B 728, 256 (2014) [arXiv:1309.6888 [hep-ph]].

[68] A. G. Akeroyd and H. Sugiyama, Phys. Rev. D 84, 035010 (2011) arXiv:1105.2209 [hep-ph]].

[69] J. Alwall, M. Herquet, F. Maltoni, O. Mattelaer, and T. Stelzer, JHEP 1106, 128 (2011) arXiv:1106.0522 [hepph]]; J. Alwall et al., JHEP 1407, 079 (2014) arXiv:1405.0301 [hep-ph]].

[70] T. Sjostrand, S. Mrenna, and P. Z. Skands, JHEP 0605, 026 (2006) [hep-ph/0603175].

[71] S. Ovyn, X. Rouby, and V. Lemaitre, arXiv:0903.2225 [hep-ph]; J. de Favereau et al. [DELPHES 3 Collaboration], JHEP 1402, 057 (2014) [arXiv:1307.6346 [hep-ex]].

[72] E. Conte, B. Fuks, and G. Serret, Comput. Phys. Commun. 184, 222 (2013) [arXiv:1206.1599 [hep-ph]].

[73] G. Aad et al. [ATLAS Collaboration], arXiv:0901.0512 [hep-ex].

[74] S. Dittmaier et al. [LHC Higgs Cross Section Working Group Collaboration], arXiv:1101.0593 [hep-ph].

[75] G. Cowan, K. Cranmer, E. Gross, and O. Vitells, Eur. Phys. J. C 71, 1554 (2011) [Erratum-ibid. C 73, 2501 (2013)] arXiv:1007.1727 [physics.data-an]].

[76] J. M. Campbell and R. K. Ellis, Phys. Rev. D 62, 114012 (2000) [hep-ph/0006304].

[77] M. Cacciari, S. Frixione, M. L. Mangano, P. Nason, and G. Ridolfi, JHEP 0809, 127 (2008) arXiv:0804.2800 [hep-ph]]; P. Bärnreuther, M. Czakon, and A. Mitov, Phys. Rev. Lett. 109, 132001 (2012) arXiv:1204.5201 [hep-ph]]. 